NIST Advanced Manufacturing Series 200-1

\title{
Design and Configuration of the Smart Manufacturing Systems Test Bed
}

Thomas Hedberg, Jr.

Moneer Helu 


\section{NIST Advanced Manufacturing Series 200-1}

\section{Design and Configuration of the Smart Manufacturing Systems Test Bed}

Thomas Hedberg, Jr.

Moneer Helu

Systems Integration Division

Engineering Laboratory

This publication is available free of charge from: https://doi.org/10.6028/NIST.AMS.200-1

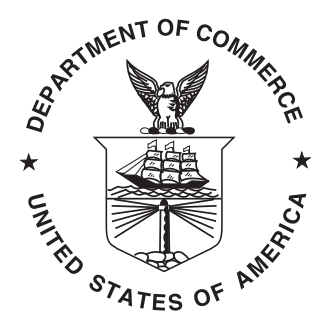

U.S. Department of Commerce Wilbur L. Ross, Jr., Secretary

National Institute of Standards and Technology Walter Copan, NIST Director and Under Secretary of Commerce for Standards and Technology 


\section{Table of Contents}

1 Introduction 1

1.1 Purpose 1

1.2 Description of the SMS Test Bed 1

1.3 Disclaimer 2

1.4 Overview 2

2 Current Resources $\quad 2$

2.1 Computer-aided technologies (CAx) Lab 2

2.2 Manufacturing Lab 4

2.3 Web Services $\quad 4$

3 Future Resources $\quad 10$

$\begin{array}{lll}3.1 \text { CAx Lab } & 10\end{array}$

3.2 Manufacturing Lab 10

4 Usage and Guidelines $\quad 10$

4.1 Architecture $r$

4.2 Connectivity, Deployment, and Usage $\quad 11$

4.3 Distributing Data 11

4.4 Query-able data repository (QDR) User Manual 11

4.5 Data Repositories 12

References $\quad 12$

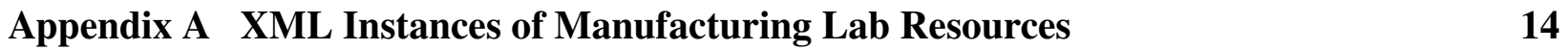




\section{List of Tables}

Table 1 Software-based resources available currently in the CAx Lab 3

Table 2 Hardware-based resources available currently in the CAx Lab

Table 3 Machine-tool resources available for fabrication activities currently in the Manufacturing Lab

Table 4 Metrology equipment resources available for digital-inspection activities currently in the Manufacturing Lab

Table 5 Available DataItem in the VDS and QDR for the GFAgie01 device 5

Table 6 Available DataItem in the VDS and QDR for the Mazak01 device 6

Table 7 Available DataItem in the VDS and QDR for the Mazak03 device 7

Table 8 Available DataItem in the VDS and QDR for the Hurco01 device 8

Table 9 Available DataItem in the VDS and QDR for the Hurco02 device 8

Table 10 Available DataItem in the VDS and QDR for the Hurco03 device 8

Table 11 Available DataItem in the VDS and QDR for the Hurco04 device 9

Table 12 Available DataItem in the VDS and QDR for the Hurco05 device 9

Table 13 Available DataItem in the VDS and QDR for the Hurco06 device 9

\section{List of Figures}

Fig. 1 Smart Manufacturing Systems (SMS) Test Bed Components 1

\section{List of Listings}

Listing A.1 MTConnect Probe Listing for the Manufacturing Lab Resources in the SMS Test Bed 


\title{
List of Acronyms
}

\author{
API Application Programming Interface. 12 \\ ASCII American Standard Code for Information Interchange. 12 \\ CAD computer-aided design. 2 \\ CAI computer-aided inspection. 2 \\ CAM computer-aided manufacturing. 2 \\ CAx computer-aided technologies. i, ii, 1-4, 10, 12 \\ CMM coordinate-measurement machine. 3, 4 \\ CNC computer numerical control. 3, 4 \\ CSV comma separated value. 12 \\ FTO Fabrication Technology Office. 4 \\ JSON JavaScript object notation. 12
}

MDCS Material Data Curation System. 4, 12

NIST National Institute of Standards and Technology. 1, 2, 4, 11, 12

NoSQL Non-Structured Query Language. 12

PDM product-data management. 2, 3

QDR query-able data repository. i, 4, 11, 12

QIF Quality Information Framework. 10

REST representational state transfer. 12

SMEs small-and-medium enterprises. 10

SMS Smart Manufacturing Systems. ii, 1, 2, 4, 10-12, 14

SRS software requirements specification. 11 
TDP technical-data packages. 4, 12

V\&V verification \& validation. 2

VDS volatile-data stream. 4, 5, 11, 12

XML Extensible Markup Language. 12

XSD XML Schema Definition. 12 


\section{Introduction}

\subsection{Purpose}

The purpose of this document is to describe the design and configuration of the National Institute of Standards and Technology (NIST) Smart Manufacturing Systems (SMS) Test Bed. Prospective developers, technical-assessment personnel, researchers, and interested end-users are the intended audience of this document. This document will provide a high-level overview of the SMS Test Bed and direct the reader to additional documentation to support fully using and replicating the SMS Test Bed.

\subsection{Description of the SMS Test Bed}

The goal of the SMS Test Bed is to extend existing production-focused concepts by designing and architecting a test bed that enables smart manufacturing research and development across the product lifecycle. This process should highlight the challenges and requirements for introducing cyber-physical infrastructure in manufacturing, as well as create opportunities to provide a tangible source of data that other researchers may use to develop and validate smart-manufacturing technologies. It also requires the integration of systems from each product-lifecycle stage so that information may flow between design, fabrication, and inspection.

The SMS Test Bed enables the development of technologies and standards that create a "digital thread" to exchange information, including product design and quality and equipment performance and health, across the product lifecycle. These efforts complement existing research and development aimed at reducing cycle time, ensuring first-pass success, and improving the overall performance of the product design and manufacturing process.

The SMS Test Bed is comprised of three major components (see Figure 1): (1) Computer-aided technologies (CAx) Lab, (2) Manufacturing Lab, and (3) Data publication web services.

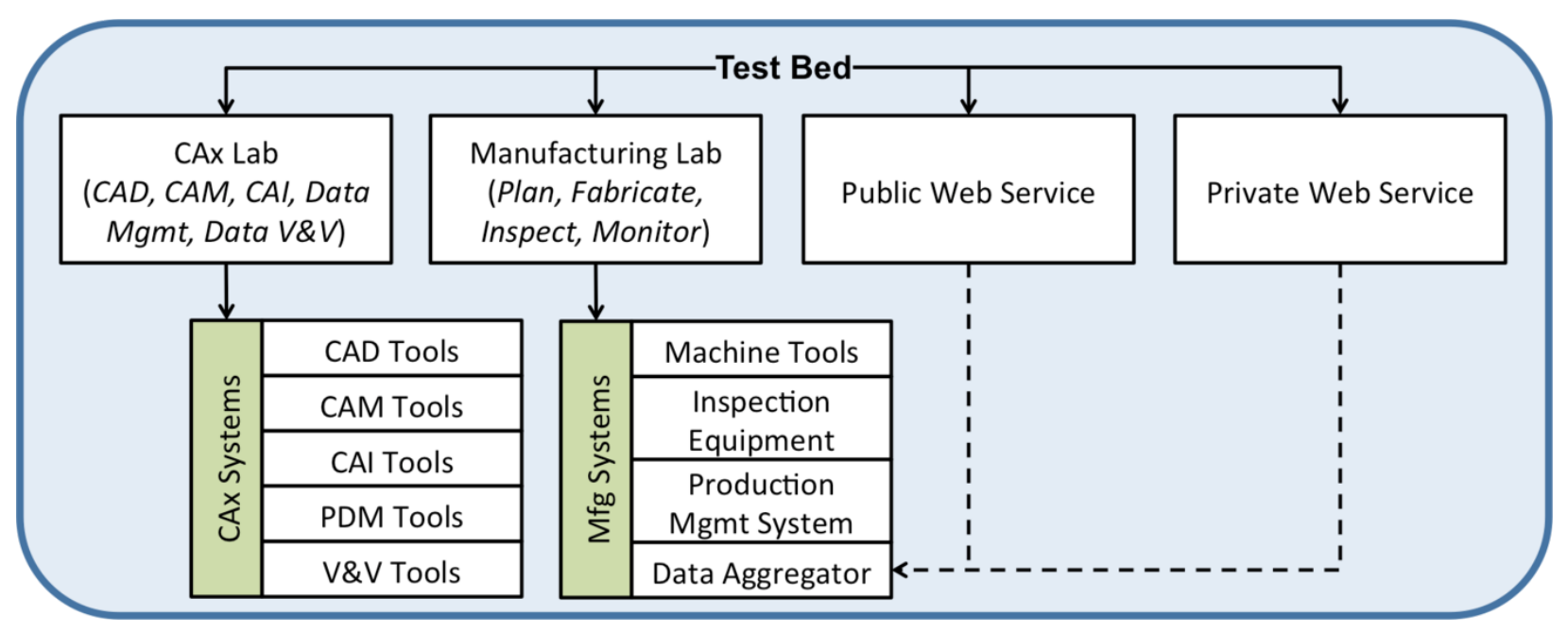

Fig. 1. SMS Test Bed Components

This publication freely available at: https://doi.org/10.6028/NIST.AMS.200-1 


\subsection{Disclaimer}

This work is an official contribution of the NIST and not subject to copyright in the United States. Certain commercial systems are identified in this paper. Such identification does not imply recommendation or endorsement by NIST. Nor does it imply that the products identified are necessarily the best available for the purpose.

\subsection{Overview}

The remainder of this document contains a description of the current resources (Section 2) and the planned or future resources (Section 3) of the SMS Test Bed. In closing, this document will list citations and additional references (Section 4) for the SMS Test Bed, which will provide guidance on using and replicating the SMS Test bed.

\section{Current Resources}

\subsection{CAx Lab}

The CAx Lab contains several computer-aided technology tools from the computer-aided design (CAD), computer-aided manufacturing (CAM), computer-aided inspection (CAI), productdata management (PDM), and verification \& validation $(\mathrm{V} \& \mathrm{~V})$ domains. Uses of the CAx Lab include, but are not limited to, developing test cases for conformance validation to standards, generating CAD models for experiments, and testing cyber-physical connections across the product lifecycle. Table 1 and Table 2 list the current software and hardware resources, respectively, available within the CAx Lab. 
Table 1. Software-based resources available currently in the CAx Lab

\begin{tabular}{llll}
\hline Name & Description & Category & Manufacturer \\
\hline Anark Core WS & PDF/PRC generator & DEX & Anark Corp \\
CADIQ & Model validation & V\&V & ITI \\
Creo & Parametric modeling system & CAD & PTC \\
ESPRIT CAM & Computer numerical control & CAM & DP Technology Corp \\
& (CNC) programing tool & & \\
IDA-STEP & STEP file viewer & CAD & LKSoftWare GmbH \\
Innovator & Open-source PDM tool & PDM & Aras Corp \\
MasterCAM & CNC programing tool & CAM & CNC Software \\
mCosmos & On-line and off-line coordinate- & CAI & Mitutoyo \\
& measurement machine (CMM) & & \\
MeasureLink & execution & & \\
MiCat Planner & CMM planning software & CAI & Mitutoyo \\
NiFi & Data-flow system & PDM & Apache Software Foundation \\
NX & Parametric modeling system & CAD & Siemens PLM \\
Solidworks & Parametric modeling system & CAD & Dassault Systems \\
Solidworks MBD & Model-based generation tool & CAD & Dassault Systems \\
\hline
\end{tabular}

Legend: $\mathrm{CAD}=$ computer-aided design, $\mathrm{CAM}=$ computer-aided manufacturing, $\mathrm{CAI}=$ computer-aided inspection, $\mathrm{DEX}=$ data exchange, $\mathrm{PDM}=$ product-data management, $\mathrm{V} \& \mathrm{~V}=$ verification $\&$ validation

Table 2. Hardware-based resources available currently in the CAx Lab

\begin{tabular}{llllll}
\hline Purpose & Processor & RAM & Video & HHD & OS \\
\hline General Use & Intel, i5-3550S, 4-core, 3 GHz & $4 \mathrm{~GB}, 1600 \mathrm{MHz}$ & Intel HD Graphics 2500 & $250 \mathrm{~GB}, 7.2 \mathrm{~K}$ & Windows \\
Workstation & Intel, E5-2630, 6-core, 2.6 GHz & $4 \times 4 \mathrm{~GB}(16 \mathrm{~GB}), 1600 \mathrm{MHz}$ & AMD FirePro V5900 (2GB) & $1 \mathrm{~TB}, 7.2 \mathrm{~K}$ & Windows \\
Workstation & Intel, E5-2630, 6-core, 2.6 GHz & $4 \times 4 \mathrm{~GB}(16 \mathrm{~GB}), 1600 \mathrm{MHz}$ & AMD FirePro V5900 (2GB) $1 \mathrm{~TB}, 7.2 \mathrm{~K}$ & Windows \\
Workstation & Intel, E5-2609, 4-core, $2.4 \mathrm{GHz}$ & $4 \times 4 \mathrm{~GB}(16 \mathrm{~GB}), 1600 \mathrm{MHz}$ & AMD FirePro V5900 (2GB) & $1 \mathrm{~TB}, 7.2 \mathrm{~K}$ & Windows \\
Workstation & Intel, E5-2609, 4-core, $2.4 \mathrm{GHz}$ & $4 \times 4 \mathrm{~GB}(16 \mathrm{~GB}), 1600 \mathrm{MHz}$ & AMD FirePro V5900 (2GB) & $1 \mathrm{~TB}, 7.2 \mathrm{~K}$ & Windows \\
License Server & Intel, i5-3550S, 4-core, $3 \mathrm{GHz}$ & $4 \mathrm{~GB}, 1600 \mathrm{MHz}$ & Intel HD Graphics 2500 & $250 \mathrm{~GB}, 7.2 \mathrm{~K}$ & Windows \\
Server & 2x Intel, E5-2670, 12-core, $2.3 \mathrm{GHz}$ & $2 \times 16 \mathrm{~GB}(32 \mathrm{~GB}), 2133 \mathrm{MHz}$ & on-board & $4 \mathrm{~TB}, 7.2 \mathrm{k}$ & Linux \\
Server & 2x Intel, E5-2670, 12-core, $2.3 \mathrm{GHz}$ & $2 \times 16 \mathrm{~GB}(32 \mathrm{~GB}), 2133 \mathrm{MHz}$ & on-board & $8 \mathrm{~T}$ 1TB, 7.2k & Windows \\
\hline
\end{tabular}




\subsection{Manufacturing Lab}

The Manufacturing Lab, in partnership with the NIST Fabrication Technology Office (FTO), mimics the configuration of a contract-manufacturing shop. The Manufacturing Lab contains several fabrication machine tools (e.g., CNC milling, CNC turning) and metrology / inspection equipment (e.g., CMM, digital micrometers). Table 3 and Table 4 list the current machine tools and metrology equipment resources, respectively, available within the Manufacturing Lab.

Table 3. Machine-tool resources available for fabrication activities currently in the Manufacturing Lab

\begin{tabular}{lllll}
\hline Device & UUID & Manufacturer & Model & Type \\
\hline GFAgie01 & mtc_adapter001 & Agie Charmilles & Micron HPM600U & 5-axis milling center \\
Mazak01 & mtc_adapter002 & Mazak & Integrex 100-IV & Mill-turn center \\
Mazak03 & mtc_adapter004 & Mazak & QuickTurn Nexus 300 & Turning center \\
Hurco01 & mtc_adapter005 & Hurco & VMX 24 & 3-axis milling center \\
Hurco02 & mtc_adapter006 & Hurco & VMX 24 & 3-axis milling center \\
Hurco03 & mtc_adapter007 & Hurco & VMX 24 & 3-axis milling center \\
Hurco04 & mtc_adapter008 & Hurco & VMX 24 & 3-axis milling center \\
Hurco05 & mtc_adapter009 & Hurco & VMX 42 & 3-axis milling center \\
Hurco06 & mtc_adapter010 & Hurco & VMX 64 & 3-axis milling center \\
\hline
\end{tabular}

Table 4. Metrology equipment resources available for digital-inspection activities currently in the Manufacturing Lab

\begin{tabular}{lllll}
\hline Device & UUID & Manufacturer & Model & Type \\
\hline Mitutoyo01 & mtc_met_adapter001 & Mitutoyo & BHN 710 & 5-axis CMM \\
Mitutoyo02 & mtc_met_adapter002 & Mitutoyo & 293-130 & Micrometers \\
Mitutoyo03 & mtc_met_adapter003 & Mitutoyo & SJ-210 & Surface profilometer \\
\hline
\end{tabular}

\subsection{Web Services}

Data is collected from the Manufacturing Lab using the MTConnect standard [1]. That data is aggregated and published internally and externally of NIST via web services. Three channels of data dissemination are available from the SMS Test Bed: (1) a volatile-data stream (VDS) using an MTConnect agent, (2) a query-able data repository (QDR) using the NIST Material Data Curation System (MDCS), and (3) pre-compiled technical-data packages (TDP) that include a collection of CAx Lab data and associated Manufacturing Lab data.

Table 5 through Table 13 provide the available DataItems in both the VDS and QDR for each device listed in Table 3. Each DataItem is listed by its Id, Name, Category, Type, Sub Type, and Units. Any DataItem that has a "x:" prefixing its Type and/or Sub Type is a machine-specific 
extension of the MTConnect Data Dictionary. Refer to Part 2 of the MTConnect standard [2] for more information about DataItem, Category, Type, and Sub Type. The raw probe output ${ }^{1}$ from MTconnect agent in the VDS is provided in Appendix A.

Table 5. Available DataItem in the VDS and QDR for the GFAgie01 device

\begin{tabular}{llllll}
\hline Id & Name & Category & Type & Sub Type & Units \\
\hline GFAgie01-dtop_1 & avail & EVENT & AVAILABILITY & null & null \\
GFAgie01-dtop_2 & estop & EVENT & EMERGENCY_STOP & null & null \\
GFAgie01-dtop_3 & system & CONDITION & SYSTEM & null & null \\
GFAgie01-X_2 & Xposition & SAMPLE & POSITION & ACTUAL & MILLIMETER \\
GFAgie01-Y_2 & Yposition & SAMPLE & POSITION & ACTUAL & MILLIMETER \\
GFAgie01-Z_2 & Zposition & SAMPLE & POSITION & ACTUAL & MILLIMETER \\
GFAgie01-C_2 & Cposition & SAMPLE & ANGLE & ACTUAL & DEGREE \\
GFAgie01-A_2 & Aposition & SAMPLE & ANGLE & ACTUAL & DEGREE \\
GFAgie01-controller_basic_2 & Fovr & EVENT & PATH_FEEDRATE_OVERRIDE & null & PERCENT \\
GFAgie01-controller_basic_3 & Sovr & EVENT & ROTARY_VELOCITY_OVERRIDE & null & PERCENT \\
GFAgie01-controller_basic_4 & servo & CONDITION & ACTUATOR & null & null \\
GFAgie01-controller_basic_5 & comms & CONDITION & COMMUNICATIONS & null & null \\
GFAgie01-controller_basic_6 & pneumatic & CONDITION & COMMUNICATIONS & null & null \\
GFAgie01-controller_basic_7 & hydraulic & CONDITION & COMMUNICATIONS & null & null \\
GFAgie01-controller_basic_8 & logic & CONDITION & LOGIC_PROGRAM & null & null \\
GFAgie01-controller_basic_9 & motion & CONDITION & MOTION_PROGRAM & null & null \\
GFAgie01-controller_basic_10 & cnc_temp & CONDITION & TEMPERATURE & null & null \\
GFAgie01-path_basic_2 & execution & EVENT & EXECUTION & null & null \\
GFAgie01-path_basic_3 & mode & EVENT & CONTROLLER_MODE & null & null \\
GFAgie01-path_basic_4 & program & EVENT & PROGRAM & null & null \\
GFAgie01-path_basic_5 & line & EVENT & LINE & null & null \\
GFAgie01-path_basic_6 & move & EVENT & X:MOTION & null & null \\
GFAgie01-path_basic_7 & path_pos & SAMPLE & PATH_POSITION & null & MILLIMETER_3D \\
\hline
\end{tabular}

${ }^{1}$ The real-time probe out is available at https://smstestbed.nist.gov/vds/probe 
Table 6. Available DataItem in the VDS and QDR for the Mazak01 device

\begin{tabular}{|c|c|c|c|c|c|}
\hline Id & Name & Category & Type & Sub Type & Units \\
\hline Mazak01-dtop_1 & avail & EVENT & AVAILABILITY & null & null \\
\hline Mazak01-base_1 & servo_cond & CONDITION & ACTUATOR & null & null \\
\hline Mazak01-X_1 & Xabs & SAMPLE & POSITION & ACTUAL & MILLIMETER \\
\hline Mazak01-X_2 & Xtravel & CONDITION & POSITION & null & null \\
\hline Mazak01-X_3 & Xload & SAMPLE & LOAD & null & PERCENT \\
\hline Mazak01-X_4 & Xfrt & SAMPLE & AXIS_FEEDRATE & null & MILLIMETER/SECOND \\
\hline Mazak01-Y_1 & Yabs & SAMPLE & POSITION & ACTUAL & MILLIMETER \\
\hline Mazak01-Y_2 & Ytravel & CONDITION & POSITION & null & null \\
\hline Mazak01-Y_3 & Yload & SAMPLE & LOAD & null & PERCENT \\
\hline Mazak01-Y_4 & Yfrt & SAMPLE & AXIS_FEEDRATE & null & MILLIMETER/SECOND \\
\hline Mazak01-Z_1 & Zabs & SAMPLE & POSITION & ACTUAL & MILLIMETER \\
\hline Mazak01-Z_2 & Ztravel & CONDITION & POSITION & null & null \\
\hline Mazak01-Z_3 & Zload & SAMPLE & LOAD & null & PERCENT \\
\hline Mazak01-Z_4 & Zfrt & SAMPLE & AXIS_FEEDRATE & null & MILLIMETER/SECOND \\
\hline Mazak01-B_1 & Bload & SAMPLE & LOAD & null & PERCENT \\
\hline Mazak01-B_2 & Bfrt & SAMPLE & ANGULAR_VELOCITY & null & DEGREE/SECOND \\
\hline Mazak01-B_3 & Btravel & CONDITION & ANGLE & null & null \\
\hline Mazak01-B_4 & Bdeg & SAMPLE & ANGLE & ACTUAL & DEGREE \\
\hline Mazak01-C_1 & Cload & SAMPLE & LOAD & null & PERCENT \\
\hline Mazak01-C_2 & Sload & SAMPLE & LOAD & null & PERCENT \\
\hline Mazak01-C_3 & Ctravel & CONDITION & ANGLE & null & null \\
\hline Mazak01-C_4 & Cfrt & SAMPLE & ANGULAR_VELOCITY & null & DEGREE/SECOND \\
\hline Mazak01-C_5 & Srpm & SAMPLE & ROTARY_VELOCITY & ACTUAL & REVOLUTION/MINUTE \\
\hline Mazak01-C_6 & Sovr & EVENT & ROTARY_VELOCITY_OVERRIDE & null & PERCENT \\
\hline Mazak01-C_7 & Stemp & SAMPLE & TEMPERATURE & null & CELSIUS \\
\hline Mazak01-C_8 & Cdeg & SAMPLE & ANGLE & ACTUAL & DEGREE \\
\hline Mazak01-C_9 & Sload_cond & CONDITION & LOAD & null & null \\
\hline Mazak01-C_10 & Stemp_cond & CONDITION & TEMPERATURE & null & null \\
\hline Mazak01-C2_1 & S2load & SAMPLE & LOAD & null & PERCENT \\
\hline Mazak01-C2_2 & S2rpm & SAMPLE & ROTARY_VELOCITY & ACTUAL & REVOLUTION/MINUTE \\
\hline Mazak01-C2_3 & S2temp & SAMPLE & TEMPERATURE & null & CELSIUS \\
\hline Mazak01-C2_4 & S2load_cond & CONDITION & LOAD & null & null \\
\hline Mazak01-C2_5 & S2temp_cond & CONDITION & TEMPERATURE & null & null \\
\hline Mazak01-controller_1 & comms_cond & CONDITION & COMMUNICATIONS & null & null \\
\hline Mazak01-controller_2 & logic_cond & CONDITION & LOGIC_PROGRAM & null & null \\
\hline Mazak01-controller_3 & system_cond & CONDITION & SYSTEM & null & null \\
\hline Mazak01-controller_4 & estop & EVENT & EMERGENCY_STOP & null & null \\
\hline Mazak01-path_1 & program & EVENT & PROGRAM & null & null \\
\hline Mazak01-path_2 & subprogram & EVENT & PROGRAM & $\mathrm{x}:$ SUB & null \\
\hline Mazak01-path_3 & line & EVENT & LINE & null & null \\
\hline Mazak01-path_4 & unitNum & EVENT & $\mathrm{x}: \mathrm{UNIT}$ & null & null \\
\hline Mazak01-path_5 & sequenceNum & EVENT & x:SEQUENCE_NUMBER & null & null \\
\hline Mazak01-path_6 & PartCountAct & EVENT & PART_COUNT & null & null \\
\hline Mazak01-path_7 & Fact & SAMPLE & PATH_FEEDRATE & ACTUAL & MILLIMETER/SECOND \\
\hline Mazak01-path_8 & Frapidovr & EVENT & PATH_FEEDRATE_OVERRIDE & RAPID & PERCENT \\
\hline Mazak01-path_9 & Fovr & EVENT & PATH_FEEDRATE_OVERRIDE & PROGRAMMED & PERCENT \\
\hline Mazak01-path_10 & Tool_number & EVENT & TOOL_NUMBER & null & null \\
\hline Mazak01-path_11 & Tool_group & EVENT & x:TOOL_GROUP & null & null \\
\hline Mazak01-path_12 & Tool_suffix & EVENT & x:TOOL_SUFFIX & null & null \\
\hline Mazak01-path_13 & execution & EVENT & EXECUTION & null & null \\
\hline Mazak01-path_14 & mode & EVENT & CONTROLLER_MODE & null & null \\
\hline Mazak01-path_15 & program_cmt & EVENT & x:PROGRAM_COMMENT & null & null \\
\hline Mazak01-path_16 & subprogram_cmt & EVENT & x:PROGRAM_COMMENT & $\mathrm{x}:$ SUB & null \\
\hline Mazak01-path_17 & auto_time & SAMPLE & ACCUMULATED_TIME & $\mathrm{x}:$ AUTO & null \\
\hline Mazak01-path_18 & total_time & SAMPLE & ACCUMULATED_TIME & x:TOTAL & null \\
\hline Mazak01-path_19 & cut_time & SAMPLE & ACCUMULATED_TIME & $\mathrm{x}:$ CUT & null \\
\hline Mazak01-path_20 & motion_cond & CONDITION & MOTION_PROGRAM & null & null \\
\hline Mazak01-path_21 & path_system & CONDITION & SYSTEM & null & null \\
\hline Mazak01-electric_1 & power & EVENT & POWER_STATE & null & null \\
\hline Mazak01-electric_2 & electric_temp & CONDITION & TEMPERATURE & null & null \\
\hline Mazak01-hydraulic_1 & hydra_cond & CONDITION & PRESSURE & null & null \\
\hline Mazak01-coolant_1 & coolant_pres & CONDITION & PRESSURE & null & null \\
\hline Mazak01-coolant_2 & coolant_temp & CONDITION & TEMPERATURE & null & null \\
\hline Mazak01-coolant_3 & coolant_level & CONDITION & LEVEL & null & null \\
\hline Mazak01-pneumatic_1 & pneu_cond & CONDITION & PRESSURE & null & null \\
\hline
\end{tabular}


Table 7. Available DataItem in the VDS and QDR for the Mazak03 device

\begin{tabular}{|c|c|c|c|c|c|}
\hline $\mathrm{Id}$ & Name & Category & Type & Sub Type & Units \\
\hline Mazak03-dtop_1 & avail & EVENT & AVAILABILITY & null & null \\
\hline Mazak03-base_2 & servo_cond & CONDITION & ACTUATOR & null & null \\
\hline Mazak03-X_2 & Xabs & SAMPLE & POSITION & ACTUAL & MILLIMETER \\
\hline Mazak03-X_3 & Xtravel & CONDITION & POSITION & null & null \\
\hline Mazak03-X_4 & Xload & SAMPLE & LOAD & null & PERCENT \\
\hline Mazak03-X_5 & Xfrt & SAMPLE & AXIS_FEEDRATE & null & MILLIMETER/SECOND \\
\hline Mazak03-Z_2 & Zabs & SAMPLE & POSITION & ACTUAL & MILLIMETER \\
\hline Mazak03-Z_3 & Ztravel & CONDITION & POSITION & null & null \\
\hline Mazak03-Z_4 & Zload & SAMPLE & LOAD & null & PERCENT \\
\hline Mazak03-Z_5 & Zfrt & SAMPLE & AXIS_FEEDRATE & null & MILLIMETER/SECOND \\
\hline Mazak03-C_2 & Cload & SAMPLE & LOAD & null & PERCENT \\
\hline Mazak03-C_3 & Cfrt & SAMPLE & ANGULAR_VELOCITY & null & DEGREE/SECOND \\
\hline Mazak03-C_4 & Cdeg & SAMPLE & ANGLE & ACTUAL & DEGREE \\
\hline Mazak03-C_5 & Ctravel & CONDITION & ANGLE & null & null \\
\hline Mazak03-S_2 & Srpm & SAMPLE & ROTARY_VELOCITY & ACTUAL & REVOLUTION/MINUTE \\
\hline Mazak03-S_3 & S1load & SAMPLE & LOAD & null & PERCENT \\
\hline Mazak03-S_4 & Stemp & SAMPLE & TEMPERATURE & null & CELSIUS \\
\hline Mazak03-S_5 & Sload_cond & CONDITION & LOAD & null & null \\
\hline Mazak03-S_6 & Stemp_cond & CONDITION & TEMPERATURE & null & null \\
\hline Mazak03-S2_2 & S2rpm & SAMPLE & ROTARY_VELOCITY & ACTUAL & REVOLUTION/MINUTE \\
\hline Mazak03-S2_3 & S2load & SAMPLE & LOAD & null & PERCENT \\
\hline Mazak03-S2_4 & S2temp & SAMPLE & TEMPERATURE & null & CELSIUS \\
\hline Mazak03-S2_5 & S2load_cond & CONDITION & LOAD & null & null \\
\hline Mazak03-S2_6 & S2temp_cond & CONDITION & TEMPERATURE & null & null \\
\hline Mazak03-controller_2 & comms_cond & CONDITION & COMMUNICATIONS & null & null \\
\hline Mazak03-controller_3 & logic_cond & CONDITION & LOGIC_PROGRAM & null & null \\
\hline Mazak03-controller_4 & system_cond & CONDITION & SYSTEM & null & null \\
\hline Mazak03-controller_5 & pallet_num & EVENT & $\mathrm{x}$ :PALLET_NUMBER & null & null \\
\hline Mazak03-path_2 & Sovr & EVENT & ROTARY_VELOCITY_OVERRIDE & null & null \\
\hline Mazak03-path_3 & program & EVENT & PROGRAM & null & null \\
\hline Mazak03-path_4 & subprogram & EVENT & PROGRAM & $\mathrm{x}:$ SUB & null \\
\hline Mazak03-path_5 & line & EVENT & LINE & null & null \\
\hline Mazak03-path_6 & unitNum & EVENT & $\mathrm{x}: \mathrm{UNIT}$ & null & null \\
\hline Mazak03-path_95 & sequenceNum & EVENT & $\mathrm{x}:$ SEQUENCE_NUMBER & null & null \\
\hline Mazak03-path_96 & PartCountAct & EVENT & PART_COUNT & null & null \\
\hline Mazak03-path_7 & Fact & SAMPLE & PATH_FEEDRATE & ACTUAL & MILLIMETER/SECOND \\
\hline Mazak03-path_8 & Frapidovr & EVENT & PATH_FEEDRATE_OVERRIDE & RAPID & PERCENT \\
\hline Mazak03-path_9 & Fovr & EVENT & PATH_FEEDRATE_OVERRIDE & PROGRAMMED & PERCENT \\
\hline Mazak03-path_10 & execution & EVENT & EXECUTION & null & null \\
\hline Mazak03-path_11 & mode & EVENT & CONTROLLER_MODE & null & null \\
\hline Mazak03-path_12 & program_cmt & EVENT & PROGRAM_COMMENT & null & null \\
\hline Mazak03-path_13 & subprogram_cmt & EVENT & PROGRAM_COMMENT & $\mathrm{x}$ :SUB_PROGRAM & null \\
\hline Mazak03-path_14 & auto_time & SAMPLE & ACCUMULATED_TIME & $\mathrm{x}:$ AUTO & SECOND \\
\hline Mazak03-path_15 & total_time & SAMPLE & ACCUMULATED_TIME & $\mathrm{x}:$ TOTAL & SECOND \\
\hline Mazak03-path_16 & cut_time & SAMPLE & ACCUMULATED_TIME & $\mathrm{x}:$ CUT & SECOND \\
\hline Mazak03-path_17 & motion_cond & CONDITION & MOTION_PROGRAM & null & null \\
\hline Mazak03-path_18 & motion_cond2 & CONDITION & MOTION_PROGRAM & x:MOTION_PROGRAM_2 & null \\
\hline Mazak03-path_19 & motion_cond3 & CONDITION & MOTION_PROGRAM & x:MOTION_PROGRAM_3 & null \\
\hline Mazak03-path_20 & motion_cond4 & CONDITION & MOTION_PROGRAM & x:MOTION_PROGRAM_4 & null \\
\hline Mazak03-path_21 & path_system & CONDITION & SYSTEM & null & null \\
\hline Mazak03-path_22 & path_system2 & CONDITION & SYSTEM & x:SYSTEM_1 & null \\
\hline Mazak03-path_23 & path_system3 & CONDITION & SYSTEM & $\mathrm{x}:$ SYSTEM_2 & null \\
\hline Mazak03-path_24 & path_system4 & CONDITION & SYSTEM & x:SYSTEM_3 & null \\
\hline Mazak03-path_25 & Tool_number & EVENT & TOOL_ASSET_ID & null & null \\
\hline Mazak03-path_26 & Tool_group & EVENT & x:TOOL_GROUP & null & null \\
\hline Mazak03-path_27 & Tool_suffix & EVENT & x:TOOL_SUFFIX & null & null \\
\hline Mazak03-electric_2 & power & EVENT & POWER_STATE & null & null \\
\hline Mazak03-electric_3 & electric_temp & CONDITION & TEMPERATURE & null & null \\
\hline Mazak03-hydraulic_2 & hydra_cond & CONDITION & PRESSURE & null & null \\
\hline Mazak03-coolant_2 & coolant pres & CONDITION & PRESSURE & null & null \\
\hline Mazak03-coolant_3 & coolant_temp & CONDITION & TEMPERATURE & null & null \\
\hline Mazak03-coolant_4 & coolant level & CONDITION & LEVEL & null & null \\
\hline Mazak03-pneumatic_2 & pneu_cond & CONDITION & PRESSURE & null & null \\
\hline
\end{tabular}


Table 8. Available DataItem in the VDS and QDR for the Hurco01 device

\begin{tabular}{llllll}
\hline Id & Name & Category & Type & Sub Type & Units \\
\hline Hurco01-dtop_1 & avail & EVENT & AVAILABILITY & null & null \\
Hurco01-C_1 & Spindle_Speed & SAMPLE & ROTARY_VELOCITY & ACTUAL & REVOLUTION/MINUTE \\
Hurco01-C_2 & Spindle_Override & EVENT & ROTARY_VELOCITY_OVERRIDE & null & PERCENT \\
Hurco01-controller_1 & EStop_State & EVENT & EMERGENCY_STOP & null & null \\
Hurco01-path_1 & Block_Number & EVENT & LINE & null & null \\
Hurco01-path_2 & Program_Name_Running & EVENT & PROGRAM & null & null \\
Hurco01-path_3 & Program_Name_Editing & EVENT & PROGRAM_EDIT_NAME & null & null \\
Hurco01-path_4 & Part_Count & EVENT & PART_COUNT & ALL & null \\
Hurco01-path_5 & Program_Status & EVENT & EXECUTION & null & null \\
Hurco01-path_6 & Current_Tool & EVENT & TOOL_NUMBER & null & null \\
Hurco01-path_7 & Program_Runtime_Seconds & SAMPLE & ACCUMULATED_TIME & null & SECOND \\
Hurco01-path_8 & Spindle_Time & SAMPLE & ACCUMULATED_TIME & null & SECOND \\
Hurco01-path_9 & Feed_Rate & SAMPLE & PATH_FEEDRATE & ACTUAL & MILLIMETER/SECOND \\
Hurco01-path_10 & Feed_Override & SAMPLE & PATH_FEEDRATE_OVERRIDE & null & PERCENT \\
Hurco01-path_11 & Rapid_Override & SAMPLE & PATH_FEEDRATE_OVERRIDE & RAPID & PERCENT \\
\hline
\end{tabular}

Table 9. Available DataItem in the VDS and QDR for the Hurco02 device

\begin{tabular}{llllll}
\hline Id & Name & Category & Type & Sub Type & Units \\
\hline Hurco02-dtop_1 & avail & EVENT & AVAILABILITY & null & null \\
Hurco02-C_1 & Spindle_Speed & SAMPLE & ROTARY_VELOCITY & ACTUAL & REVOLUTION/MINUTE \\
Hurco02-C_2 & Spindle_Override & EVENT & ROTARY_VELOCITY_OVERRIDE & null & PERCENT \\
Hurco02-controller_1 & EStop_State & EVENT & EMERGENCY_STOP & null & null \\
Hurco02-path_1 & Block_Number & EVENT & LINE & null & null \\
Hurco02-path_2 & Program_Name_Running & EVENT & PROGRAM & null & null \\
Hurco02-path_3 & Program_Name_Editing & EVENT & PROGRAM_EDIT_NAME & null & null \\
Hurco02-path_4 & Part_Count & EVENT & PART_COUNT & ALL & null \\
Hurco02-path_5 & Program_Status & EVENT & EXECUTION & null & null \\
Hurco02-path_6 & Current_Tool & EVENT & TOOL_NUMBER & null & null \\
Hurco02-path_7 & Program_Runtime_Seconds & SAMPLE & ACCUMULATED_TIME & null & SECOND \\
Hurco02-path_8 & Spindle_Time & SAMPLE & ACCUMULATED_TIME & null & SECOND \\
Hurco02-path_9 & Feed_Rate & SAMPLE & PATH_FEEDRATE & ACTUAL & MILLIMETER/SECOND \\
Hurco02-pat__10 & Feed_Override & SAMPLE & PATH_FEEDRATE_OVERRIDE & null & PERCENT \\
Hurco02-path_11 & Rapid_Override & SAMPLE & PATH_FEEDRATE_OVERRIDE & RAPID & PERCENT \\
\hline
\end{tabular}

Table 10. Available DataItem in the VDS and QDR for the Hurco03 device

\begin{tabular}{llllll}
\hline Id & Name & Category & Type & Sub Type & Units \\
\hline Hurco03-dtop_1 & avail & EVENT & AVAILABILITY & null & null \\
Hurco03-C_1 & Spindle_Speed & SAMPLE & ROTARY_VELOCITY & ACTUAL & REVOLUTION/MINUTE \\
Hurco03-C_2 & Spindle_Override & EVENT & ROTARY_VELOCITY_OVERRIDE & null & PERCENT \\
Hurco03-controller_1 & EStop_State & EVENT & EMERGENCY_STOP & null & null \\
Hurco03-path_1 & Block_Number & EVENT & LINE & null & null \\
Hurco03-path_2 & Program_Name_Running & EVENT & PROGRAM & null & null \\
Hurco03-path_3 & Program_Name_Editing & EVENT & PROGRAM_EDIT_NAME & null & null \\
Hurco03-path_4 & Part_Count & EVENT & PART_COUNT & ALL & null \\
Hurco03-path_5 & Program_Status & EVENT & EXECUTION & null & null \\
Hurco03-path_6 & Current_Tool & EVENT & TOOL_NUMBER & null & null \\
Hurco03-path_7 & Program_Runtime_Seconds & SAMPLE & ACCUMULATED_TIME & null & SECOND \\
Hurco03-path_8 & Spindle_Time & SAMPLE & ACCUMULATED_TIME & null & SECOND \\
Hurco03-path_9 & Feed_Rate & SAMPLE & PATH_FEEDRATE & ACTUAL & MILLIMETER/SECOND \\
Hurco03-path_10 & Feed_Override & SAMPLE & PATH_FEEDRATE_OVERRIDE & null & PERCENT \\
Hurco03-path_11 & Rapid_Override & SAMPLE & PATH_FEEDRATE_OVERRIDE & RAPID & PERCENT \\
\hline
\end{tabular}


Table 11. Available DataItem in the VDS and QDR for the Hurco04 device

\begin{tabular}{llllll}
\hline Id & Name & Category & Type & Sub Type & Units \\
\hline Hurco04-dtop_1 & avail & EVENT & AVAILABILITY & null & null \\
Hurco04-C_1 & Spindle_Speed & SAMPLE & ROTARY_VELOCITY & ACTUAL & REVOLUTION/MINUTE \\
Hurco04-C_2 & Spindle_Override & EVENT & ROTARY_VELOCITY_OVERRIDE & null & PERCENT \\
Hurco04-controller_1 & EStop_State & EVENT & EMERGENCY_STOP & null & null \\
Hurco04-path_1 & Block_Number & EVENT & LINE & null & null \\
Hurco04-path_2 & Program_Name_Running & EVENT & PROGRAM & null & null \\
Hurco04-path_3 & Program_Name_Editing & EVENT & PROGRAM_EDIT_NAME & null & null \\
Hurco04-path_4 & Part_Count & EVENT & PART_COUNT & ALL & null \\
Hurco04-path_5 & Program_Status & EVENT & EXECUTION & null & null \\
Hurco04-path_6 & Current_Tool & EVENT & TOOL_NUMBER & null & null \\
Hurco04-path_7 & Program_Runtime_Seconds & SAMPLE & ACCUMULATED_TIME & null & SECOND \\
Hurco04-path_8 & Spindle_Time & SAMPLE & ACCUMULATED_TIME & null & SECOND \\
Hurco04-path_9 & Feed_Rate & SAMPLE & PATH_FEEDRATE & ACTUAL & MILLIMETER/SECOND \\
Hurco04-path_10 & Feed_Override & SAMPLE & PATH_FEEDRATE_OVERRIDE & null & PERCENT \\
Hurco04-path_11 & Rapid_Override & SAMPLE & PATH_FEEDRATE_OVERRIDE & RAPID & PERCENT \\
\hline
\end{tabular}

Table 12. Available DataItem in the VDS and QDR for the Hurco05 device

\begin{tabular}{llllll}
\hline Id & Name & Category & Type & Sub Type & Units \\
\hline Hurco05-dtop_1 & avail & EVENT & AVAILABILITY & null & null \\
Hurco05-C_1 & Spindle_Speed & SAMPLE & ROTARY_VELOCITY & ACTUAL & REVOLUTION/MINUTE \\
Hurco05-C_2 & Spindle_Override & EVENT & ROTARY_VELOCITY_OVERRIDE & null & PERCENT \\
Hurco05-controller_1 & EStop_State & EVENT & EMERGENCY_STOP & null & null \\
Hurco05-path_1 & Block_Number & EVENT & LINE & null & null \\
Hurco05-path_2 & Program_Name_Running & EVENT & PROGRAM & null & null \\
Hurco05-path_3 & Program_Name_Editing & EVENT & PROGRAM_EDIT_NAME & null & null \\
Hurco05-path_4 & Part_Count & EVENT & PART_COUNT & ALL & null \\
Hurco05-path_5 & Program_Status & EVENT & EXECUTION & null & null \\
Hurco05-path_6 & Current_Tool & EVENT & TOOL_NUMBER & null & null \\
Hurco05-path_7 & Program_Runtime_Seconds & SAMPLE & ACCUMULATED_TIME & null & SECOND \\
Hurco05-path_8 & Spindle_Time & SAMPLE & ACCUMULATED_TIME & null & SECOND \\
Hurco05-path_9 & Feed_Rate & SAMPLE & PATH_FEEDRATE & ACTUAL & MILLIMETER/SECOND \\
Hurco05-path_10 & Feed_Override & SAMPLE & PATH_FEEDRATE_OVERRIDE & null & PERCENT \\
Hurco05-path_11 & Rapid_Override & SAMPLE & PATH_FEEDRATE_OVERRIDE & RAPID & PERCENT \\
\hline
\end{tabular}

Table 13. Available DataItem in the VDS and QDR for the Hurco06 device

\begin{tabular}{llllll}
\hline Id & Name & Category & Type & Sub Type & Units \\
\hline Hurco06-dtop_1 & avail & EVENT & AVAILABILITY & null & null \\
Hurco06-C_1 & Spindle_Speed & SAMPLE & ROTARY_VELOCITY & ACTUAL & REVOLUTION/MINUTE \\
Hurco06-C_2 & Spindle_Override & EVENT & ROTARY_VELOCITY_OVERRIDE & null & PERCENT \\
Hurco06-controller_1 & EStop_State & EVENT & EMERGENCY_STOP & null & null \\
Hurco06-path_1 & Block_Number & EVENT & LINE & null & null \\
Hurco06-path_2 & Program_Name_Running & EVENT & PROGRAM & null & null \\
Hurco06-path_3 & Program_Name_Editing & EVENT & PROGRAM_EDIT_NAME & null & null \\
Hurco06-path_4 & Part_Count & EVENT & PART_COUNT & ALL & null \\
Hurco06-path_5 & Program_Status & EVENT & EXECUTION & null & null \\
Hurco06-path_6 & Current_Tool & EVENT & TOOL_NUMBER & null & null \\
Hurco06-path_7 & Program_Runtime_Seconds & SAMPLE & ACCUMULATED_TIME & null & SECOND \\
Hurco06-path_8 & Spindle_Time & SAMPLE & ACCUMULATED_TIME & null & SECOND \\
Hurco06-path_9 & Feed_Rate & SAMPLE & PATH_FEEDRATE & ACTUAL & MILLIMETER/SECOND \\
Hurco06-path_10 & Feed_Override & SAMPLE & PATH_FEEDRATE_OVERRIDE & null & PERCENT \\
Hurco06-path_11 & Rapid_Override & SAMPLE & PATH_FEEDRATE_OVERRIDE & RAPID & PERCENT \\
\hline
\end{tabular}




\section{Future Resources}

\subsection{CAx Lab}

New, alternative, or upgraded software solutions are investigated periodically for inclusion in the CAx Lab. We strive to have a mix of open-source, low-to-mid cost, and enterprise-class solutions in the CAx Lab. No specific additions are identified at this time.

\subsection{Manufacturing Lab}

Similar to the CAx Lab, new, alternative, or upgraded hardware solutions are investigated periodically for inclusion in the Manufacturing Lab. We focus on solutions that would help both small-and-medium enterprises (SMEs) and large enterprises - specifically, how the two types of enterprises could integrated more closely. At this time, we are planning the following additions to the Manufacturing Lab resources.

- Integrating external sensors into the machine tools (see Table 3)

- Thermocouples to capture temperature of various systems in the machine tools (e.g., coolant, motors, servos)

- Power meters to capture energy usage of the machine tools

- Emerging, commercially available, all-in-one manufacturing-data-capture solutions

- Enabling automated inspection data collection from the metrology equipment (see Table 4) using Quality Information Framework (QIF) [3]

\section{Usage and Guidelines}

This section provides details on how to cite the various resources, documents, and guidelines of the SMS Test Bed. Also, a description of each resource, document, and guideline is provided with the citations. Each citation is intended to direct the reader to the important information required to use and/or duplicate the SMS Test Bed.

The current document provides the overview of the SMS Test Bed. If someone wishes to reference the SMS Test Bed in general, this document should be cited. The citation for this document is as follows.

Citation: Hedberg Jr T, Helu M (2017) Design and configuration of the smart manufacturing systems test bed. National Institute of Standards and Technology, Report NIST AMS 200-1. doi: 10.6028/NIST.AMS.200-1

\subsection{Architecture}

We prepared a paper that presents the reference four-tiered architecture of the SMS Test Bed. The architecture is designed to manage the data generated by manufacturing systems for the digital thread. The architecture provides segregated access to internal and external clients, which protects 
intellectual property and other sensitive information, and enables the fusion of manufacturing and other product lifecycle data. We have implemented the architecture in the SMS Test Bed and used it to generate knowledge and identify performance improvement opportunities that would otherwise be unobservable to a manufacturing decision maker. The citation for the architecture paper is as follows.

Citation: Helu M, Hedberg Jr T, Barnard Feeney A (2017) Reference architecture to integrate heterogeneous manufacturing systems for the digital thread. CIRP Journal of Manufacturing Science and Technology doi: 10.1016/j.cirpj.2017.04.002

\subsection{Connectivity, Deployment, and Usage}

Documentation is available that describes how to connect, deploy, and use the technologies integrated in the SMS Test Bed. This document should provide baseline guidance for replicating the SMS Test Bed in both research and production environments. Specific guidance on communication protocols, information systems, and automation is included in the documentation. The citation for the connectivity, deployment, and usage guide is as follows.

Citation: Helu M, Hedberg Jr T (2017) Connecting, deploying, and using the smart manufacturing systems test bed. National Institute of Standards and Technology, Report NIST AMS 200-2. doi: 10.6028/NIST.AMS.200-2

\subsection{Distributing Data}

We published a software requirements specification (SRS) document to describe the requirements specifications for the applications and data repository used to distribute manufacturing data from the SMS Test Bed. A software system to distribute manufacturing data via a web service is the application being defined by the SRS document. The software system contains two component applications. The first component is an application to stream time-synchronized manufacturing data, referred to as the VDS. The second component is an application for querying a database containing manufacturing data, referred to as the QDR. The citation for the SRS document is as follows.

Citation: Hedberg Jr T, Helu M, Newrock M (2017) Software requirements specification to distribute manufacturing data. National Institute of Standards and Technology, Report NIST AMS 300-2. doi: 10.6028/NIST.AMS.300-2

\subsection{QDR User Manual}

The purpose of the QDR User Manual is to provide an overview of and operational guide for the QDR, which is a data curation and database management tool. The QDR has been deployed as a web service in the NIST SMS Test Bed. The purpose of the QDR is to transform collected data into structured formats that may be shared with interested end-users. Data is inputted into 
the QDR by a system administrator in an Extensible Markup Language (XML) format. This data is organized using predefined templates encoded in XML Schema Definition (XSD) that are also used to create query templates. Data is saved within the QDR in a Non-Structured Query Language (NoSQL) database. Users can search and retrieve data from this database by specifying parameter values and search criteria using a web-based form that represents the predefined query template and performs an endpoint query through a representational state transfer (REST)ful Application Programming Interface (API) call. Retrieved data may be formatted in either American Standard Code for Information Interchange (ASCII) comma separated value (CSV), JavaScript object notation (JSON), or XML structured with a reference data schema. The citation for the QDR user manual is as follows.

Citation: Bardakoff A, Dima A, Long B, Amaral GS, Helu M, Hedberg Jr T (2017) Query-able data repository: User manual, version 1.4.0-beta3. National Institute of Standards and Technology, Report NIST AMS 200-3. doi: 10.6028/NIST.AMS.200-3

\subsection{Data Repositories}

Data is collected from the NIST SMS Test Bed's Manufacturing Lab using the MTConnect standard [1]. That data is aggregated and published internally and externally of NIST via web services. Three channels of data dissemination are available from the SMS Test Bed: (1) a VDS using an MTConnect agent, (2) a QDR using the NIST MDCS, and (3) pre-compiled TDP that include a collection of CAx Lab data and associated Manufacturing Lab data. The citations for the VDS, QDR, and TDP are as follows.

VDS Citation: Helu M, Hedberg Jr T, Barnard Feeney A, Luce M (2016, Last Accessed 6/28/2017) Volatile data stream (VDS) for the smart manufacturing systems (SMS) test bed using MTConnect, Web Page. doi: 10.18434/T4FK54. URL https://smstestbed.nist.gov/vds

QDR Citation: Hedberg Jr T, Helu M, Barnard Feeney A, Luce M (2016, Last Accessed 6/28/2017) Query-able data repository (QDR) for the smart manufacturing systems (SMS) test bed using mtconnect, Web Page. URL https://smstestbed.nist.gov/qdr

TDP Citation: Hedberg Jr T, Helu M, Barnard Feeney A (2016, Last Accessed 6/28/2017) Technical data packages (TDPs) from the smart manufacturing systems (SMS) test bed, Web Page. URL https://smstestbed.nist.gov/tdp

\section{References}

[1] MTConnect Institute (2014, Last Accessed June 2017) MTConnect Standard, Version 1.3, Part 1 - Overview and Protocol, Standard. URL http://www.mtconnect.org/ standard-documents. 
[2] MTConnect Institute (2014, Last Accessed June 2017) MTConnect Standard, Version 1.3, Part 2 - Components and Data Items, Standard. URL http://www.mtconnect.org/ standard-documents.

[3] Dimensional Metrology Standards Consortium (2014) Part 1: Overview and fundamental principles in quality information framework (QIF) - an integrated model for manufacturing quality information, Standard. URL http://qifstandards.org/.

[4] Hedberg Jr T, Helu M (2017) Design and configuration of the smart manufacturing systems test bed. National Institute of Standards and Technology, Report NIST AMS 200-1. doi: 10.6028/NIST.AMS.200-1

[5] Helu M, Hedberg Jr T, Barnard Feeney A (2017) Reference architecture to integrate heterogeneous manufacturing systems for the digital thread. CIRP Journal of Manufacturing Science and Technology doi: 10.1016/j.cirpj.2017.04.002

[6] Helu M, Hedberg Jr T (2017) Connecting, deploying, and using the smart manufacturing systems test bed. National Institute of Standards and Technology, Report NIST AMS 200-2. doi: 10.6028/NIST.AMS.200-2

[7] Hedberg Jr T, Helu M, Newrock M (2017) Software requirements specification to distribute manufacturing data. National Institute of Standards and Technology, Report NIST AMS 3002. doi: 10.6028/NIST.AMS.300-2

[8] Bardakoff A, Dima A, Long B, Amaral GS, Helu M, Hedberg Jr T (2017) Query-able data repository: User manual, version 1.4.0-beta3. National Institute of Standards and Technology, Report NIST AMS 200-3. doi: 10.6028/NIST.AMS.200-3

[9] Helu M, Hedberg Jr T, Barnard Feeney A, Luce M (2016, Last Accessed 6/28/2017) Volatile data stream (VDS) for the smart manufacturing systems (SMS) test bed using MTConnect, Web Page. doi: 10.18434/T4FK54. URL https://smstestbed.nist.gov/vds

[10] Hedberg Jr T, Helu M, Barnard Feeney A, Luce M (2016, Last Accessed 6/28/2017) Queryable data repository (QDR) for the smart manufacturing systems (SMS) test bed using mtconnect, Web Page. URL https://smstestbed.nist.gov/qdr.

[11] Hedberg Jr T, Helu M, Barnard Feeney A (2016, Last Accessed 6/28/2017) Technical data packages (TDPs) from the smart manufacturing systems (SMS) test bed, Web Page. URL https://smstestbed.nist.gov/tdp. 


\section{Appendix A. XML Instances of Manufacturing Lab Resources}

Listing A.1. MTConnect Probe Listing for the Manufacturing Lab Resources in the SMS Test Bed

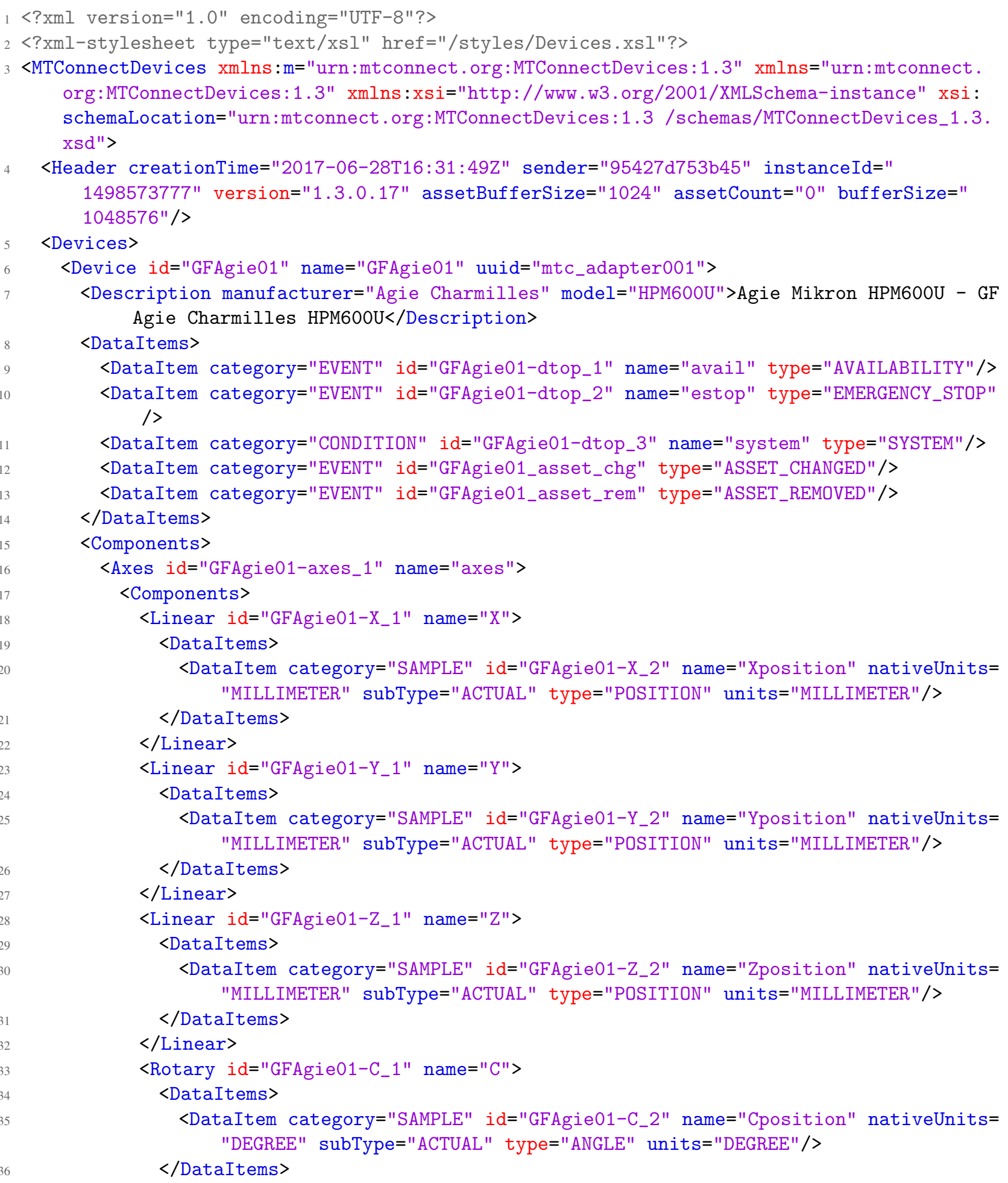

This publication freely available at: https://doi.org/10.6028/NIST.AMS.200-1 


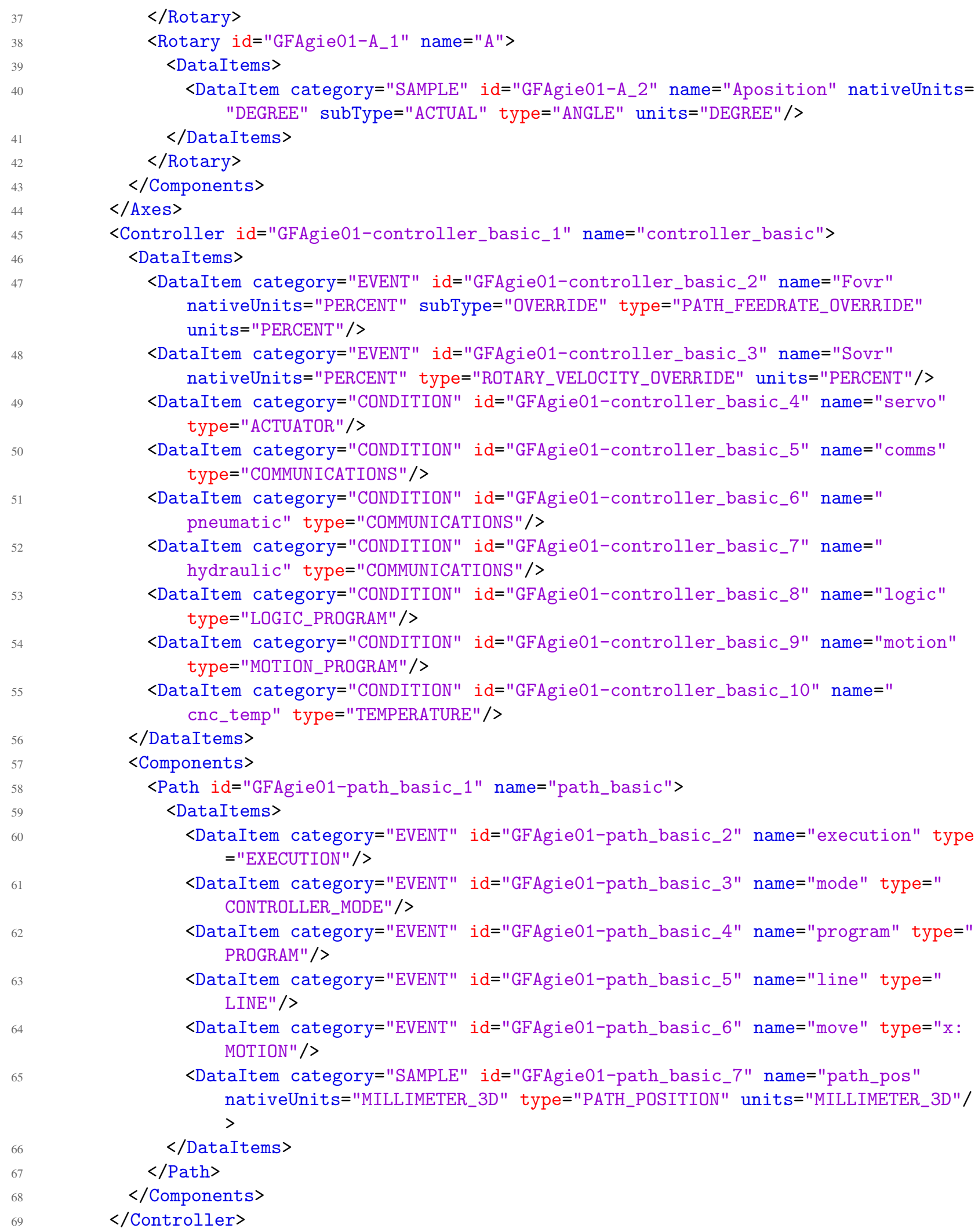




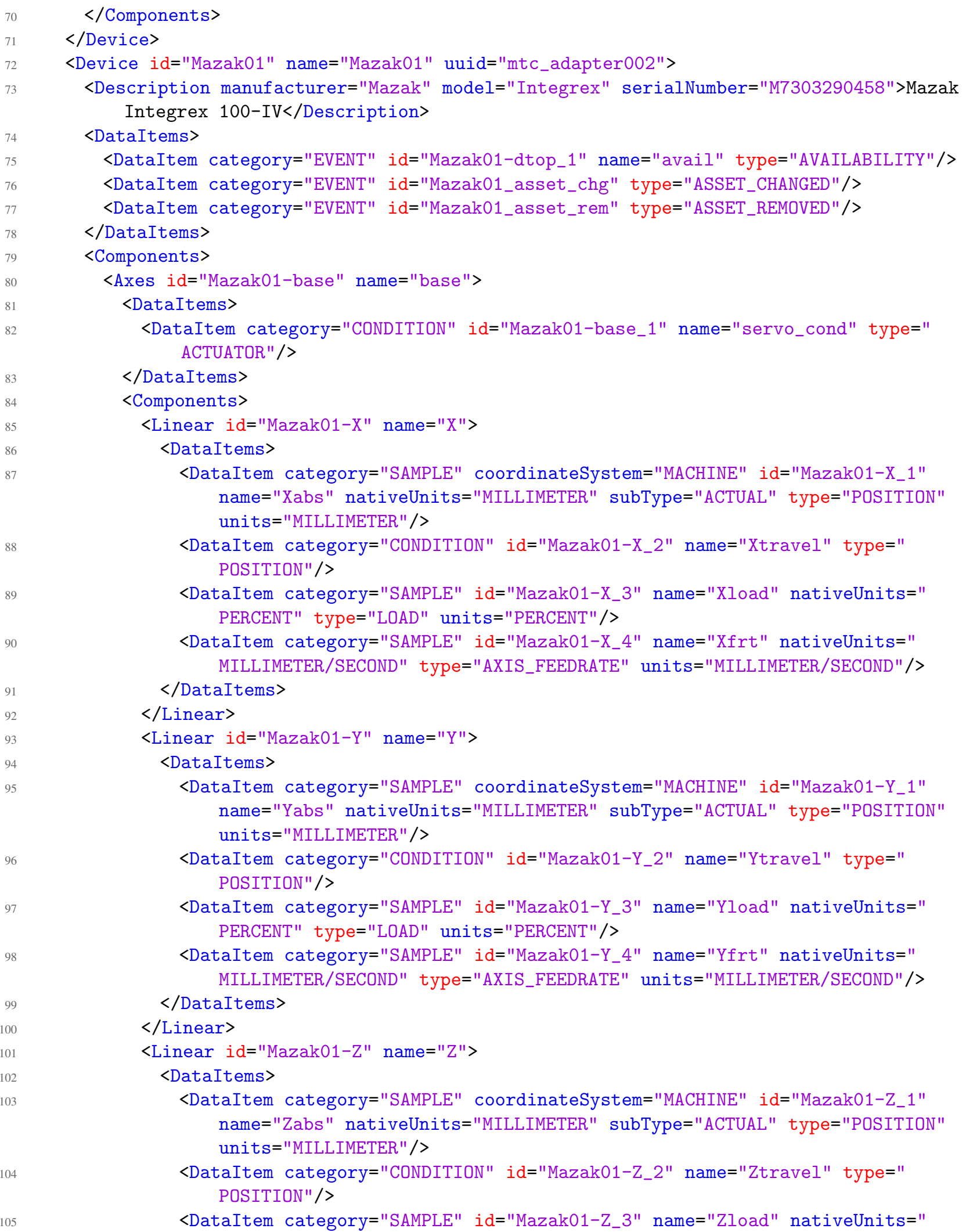


PERCENT" type="LOAD" units="PERCENT"/>

<DataItem category="SAMPLE" id="Mazak01-Z_4" name="Zfrt" nativeUnits=" MILLIMETER/SECOND" type="AXIS_FEEDRATE" units="MILLIMETER/SECOND" /> </DataItems >

$</$ Linear $>$

<Rotary id="Mazak01-B" name="B">

<DataItems>

<DataItem category="SAMPLE" id="Mazak01-B_1" name="Bload" nativeUnits=" PERCENT" type="LOAD" units $=$ "PERCENT"/>

$<$ DataItem category="SAMPLE" id="Mazak01-B_2" name="Bfrt" nativeUnits=" DEGREE/MINUTE" type="ANGULAR_VELOCITY" units="DEGREE/SECOND" />

$<$ DataItem category="CONDITION" id="Mazak01-B_3" name="Btravel" type="ANGLE" />

<DataItem category="SAMPLE" id="Mazak01-B_4" name="Bdeg" nativeUnits=" DEGREE" subType="ACTUAL" type="ANGLE" units="DEGREE" />

$</$ DataItems $>$

$</$ Rotary $>$

<Rotary id="Mazak01-C" name="C">

$<$ DataItems>

<DataItem category="SAMPLE" id="Mazak01-C_1" name="Cload" nativeUnits=" PERCENT" type="LOAD" units="PERCENT"/>

<DataItem category="SAMPLE" id="Mazak01-C_2" name="Sload" nativeUnits=" PERCENT" type="LOAD" units="PERCENT"/>

$<$ DataItem category="CONDITION" id="Mazak01-C_3" name="Ctravel" type="ANGLE" />

$<$ DataItem category="SAMPLE" id="Mazak01-C_4" name="Cfrt" nativeUnits=" DEGREE/MINUTE" type="ANGULAR_VELOCITY" units="DEGREE/SECOND" />

<DataItem category="SAMPLE" id="Mazak01-C_5" name="Srpm" nativeUnits=" REVOLUTION/MINUTE" subType="ACTUAL" type="ROTARY_VELOCITY" units=" REVOLUTION/MINUTE" />

$<$ DataItem category="EVENT" id="Mazak01-C_6" name="Sovr" nativeUnits=" PERCENT" type="ROTARY_VELOCITY_OVERRIDE" units="PERCENT" />

$<$ DataItem category="SAMPLE" id="Mazak01-C_7" name="Stemp" nativeUnits=" CELSIUS" type="TEMPERATURE" units="CELSIUS"/>

$<$ DataItem category="SAMPLE" id="Mazak01-C_8" name="Cdeg" nativeUnits=" DEGREE" subType="ACTUAL" type="ANGLE" units="DEGREE" />

<DataItem category="CONDITION" id="Mazak01-C_9" name="Sload_cond" type=" LOAD" />

$<$ DataItem category="CONDITION" id="Mazak01-C_10" name="Stemp_cond" type=" TEMPERATURE" />

</DataItems >

$</$ Rotary $>$

<Rotary id="Mazak01-C2" name="C">

$<$ DataItems $>$

<DataItem category="SAMPLE" id="Mazak01-C2_1" name="S2load" nativeUnits=" PERCENT" type="LOAD" units="PERCENT"/>

<DataItem category="SAMPLE" id="Mazak01-C2_2" name="S2rpm" nativeUnits=" REVOLUTION/MINUTE" subType="ACTUAL" type="ROTARY_VELOCITY" units=" REVOLUTION/MINUTE"/>

<DataItem category="SAMPLE" id="Mazak01-C2_3" name="S2temp" nativeUnits=" CELSIUS" type="TEMPERATURE" units="CELSIUS"/> 
$<$ DataItem category="CONDITION" id="Mazak01-C2_4" name="S2load_cond" type=" LOAD" />

$<$ DataItem category="CONDITION" id="Mazak01-C2_5" name="S2temp_cond" type=" TEMPERATURE" />

\author{
$</$ DataItems $>$ \\ $</$ Rotary $>$
}

$</$ Components $>$

$</$ Axes $>$

<Controller id="Mazak01-controller" name="controller">

$<$ DataItems $>$

$<$ DataItem category="CONDITION" id="Mazak01-controller_1" name="comms_cond" type $=$ "COMMUNICATIONS" $/>$

$<$ DataItem category="CONDITION" id="Mazak01-controller_2" name="logic_cond" type="LOGIC_PROGRAM" />

$<$ DataItem category="CONDITION" id="Mazak01-controller_3" name="system_cond" type="SYSTEM" / >

$<$ DataItem category="EVENT" id="Mazak01-controller_4" name="estop" type=" EMERGENCY_STOP"/>

$</$ DataItems>

$<$ Components $>$

$<$ Path id="Mazak01-path" name="path">

<DataItems>

$<$ DataItem category="EVENT" id="Mazak01-path_1" name="program" type="PROGRAM "/>

$<$ DataItem category="EVENT" id="Mazak01-path_2" name="subprogram" subType=" $\mathrm{x}$ :SUB" type="PROGRAM" />

<DataItem category="EVENT" id="Mazak01-path_3" name="line" type="LINE"/>

$<$ DataItem category="EVENT" id="Mazak01-path_4" name="unitNum" type=" $\mathrm{x}: \mathrm{UNIT}$ " />

$<$ DataItem category="EVENT" id="Mazak01-path_5" name="sequenceNum" type=" $\mathrm{x}$ : SEQUENCE_NUMBER"/>

$<$ DataItem category="EVENT" id="Mazak01-path_6" name="PartCountAct" type=" PART_COUNT" />

<DataItem category="SAMPLE" coordinateSystem="WORK" id="Mazak01-path_7" name="Fact" nativeUnits="FOOT/MINUTE" subType="ACTUAL" type=" PATH_FEEDRATE" units="MILLIMETER/SECOND" $/>$

<DataItem category="EVENT" id="Mazak01-path_8" name="Frapidovr" nativeUnits ="PERCENT" subType="RAPID" type="PATH_FEEDRATE_OVERRIDE" units="PERCENT $" />$

<DataItem category="EVENT" id="Mazak01-path_9" name="Fovr" nativeUnits=" PERCENT" subType="PROGRAMMED" type="PATH_FEEDRATE_OVERRIDE" units=" PERCENT" />

$<$ DataItem category="EVENT" id="Mazak01-path_10" name="Tool_number" type=" TOOL_NUMBER" />

$<$ DataItem category="EVENT" id="Mazak01-path_11" name="Tool_group" type=" $\mathrm{x}$ : TOOL_GROUP" />

$<$ DataItem category="EVENT" id="Mazak01-path_12" name="Tool_suffix" type="x: TOOL_SUFFIX" / $>$

$<$ DataItem category="EVENT" id="Mazak01-path_13" name="execution" type=" EXECUTION"/>

$<$ DataItem category="EVENT" id="Mazak01-path_14" name="mode" type=" 
CONTROLLER_MODE" />

$<$ DataItem category="EVENT" id="Mazak01-path_15" name="program_cmt" type="x: PROGRAM_COMMENT" / >

<DataItem category="EVENT" id="Mazak01-path_16" name="subprogram_cmt" subType="x:SUB" type="x:PROGRAM_COMMENT"/>

<DataItem category="SAMPLE" id="Mazak01-path_17" name="auto_time" subType=" $\mathrm{x}:$ AUTO" type="ACCUMULATED_TIME"/>

<DataItem category="SAMPLE" id="Mazak01-path_18" name="total_time" subType= "x:TOTAL" type="ACCUMULATED_TIME"/>

<DataItem category="SAMPLE" id="Mazak01-path_19" name="cut_time" subType="x :CUT" type="ACCUMULATED_TIME" />

<DataItem category="CONDITION" id="Mazak01-path_20" name="motion_cond" type $=$ "MOTION_PROGRAM" />

$<$ DataItem category="CONDITION" id="Mazak01-path_21" name="path_system" type ="SYSTEM"/>

$</$ DataItems $>$

$</$ Path $>$

$</$ Components $>$

$</$ Controller $>$

<Systems id="Mazak01-systems" name="systems">

<Components>

<Electric id="Mazak01-electric" name="electric">

<DataItems>

<DataItem category="EVENT" id="Mazak01-electric_1" name="power" type=" POWER_STATE"/>

$<$ DataItem category="CONDITION" id="Mazak01-electric_2" name="electric_temp" type="TEMPERATURE" />

$</$ DataItems $>$

$</$ Electric $>$

<Hydraulic id="Mazak01-hydraulic" name="hydraulic">

$<$ DataItems>

<DataItem category="CONDITION" id="Mazak01-hydraulic_1" name="hydra_cond" type="PRESSURE" />

$</$ DataItems $>$

$</$ Hydraulic $>$

<Coolant id="Mazak01-coolant" name="coolant">

<DataItems>

$<$ DataItem category="CONDITION" id="Mazak01-coolant_1" name="coolant_pres" type="PRESSURE" />

<DataItem category="CONDITION" id="Mazak01-coolant_2" name="coolant_temp" type="TEMPERATURE" />

<DataItem category="CONDITION" id="Mazak01-coolant_3" name="coolant_level" type="LEVEL"/>

$</$ DataItems $>$

$</$ Coolant $>$

<Pneumatic id="Mazak01-pneumatic" name="pneumatic">

<DataItems>

<DataItem category="CONDITION" id="Mazak01-pneumatic_1" name="pneu_cond" type="PRESSURE" />

$</$ DataItems $>$

$</$ Pneumatic $>$ 


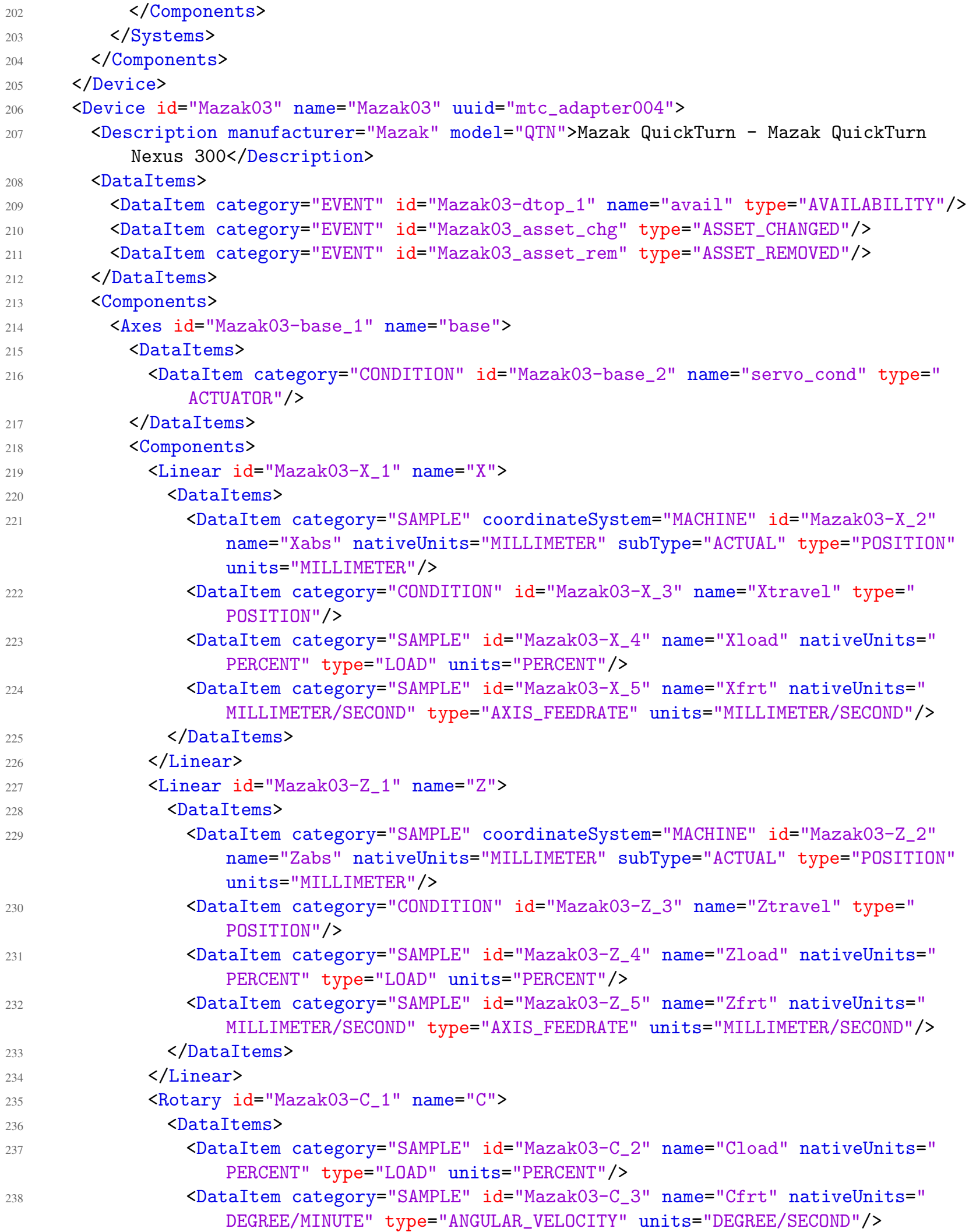


<DataItem category="SAMPLE" id="Mazak03-C_4" name="Cdeg" nativeUnits=" DEGREE" subType="ACTUAL" type="ANGLE" units="DEGREE" $/>$

$<$ DataItem category="CONDITION" id="Mazak03-C_5" name="Ctravel" type="ANGLE" />

$</$ DataItems $>$

$</$ Rotary $>$

$<$ Rotary id="Mazak03-S_1" name="S" nativeName="S">

$<$ DataItems $>$

$<$ DataItem category="SAMPLE" id="Mazak03-S_2" name="Srpm" nativeUnits=" REVOLUTION/MINUTE" subType="ACTUAL" type="ROTARY_VELOCITY" units=" REVOLUTION/MINUTE"/>

$<$ DataItem category="SAMPLE" id="Mazak03-S_3" name="S1load" nativeUnits=" PERCENT" type $=$ "LOAD" units $="$ PERCENT" $>$

$<$ Source $>$ Sload</Source $>$

$</$ DataItem $>$

$<$ DataItem category="SAMPLE" id="Mazak03-S_4" name="Stemp" nativeUnits=" CELSIUS" type $=$ "TEMPERATURE" units $="$ CELSIUS" $/>$

$<$ DataItem category="CONDITION" id="Mazak03-S_5" name="Sload_cond" type=" LOAD" />

$<$ DataItem category="CONDITION" id="Mazak03-S_6" name="Stemp_cond" type=" TEMPERATURE" / $>$

$<$ DataItems $>$

$</$ Rotary $>$

$<$ Rotary id="Mazak03-S2_1" name="S2" nativeName="S2">

$<$ DataItems>

$<$ DataItem category="SAMPLE" id="Mazak03-S2_2" name="S2rpm" nativeUnits=" REVOLUTION/MINUTE" subType="ACTUAL" type="ROTARY_VELOCITY" units=" REVOLUTION/MINUTE" />

<DataItem category="SAMPLE" id="Mazak03-S2_3" name="S2load" nativeUnits=" PERCENT" type $=$ "LOAD" units $="$ PERCENT" $>$

$<$ Source $>$ S2load</Source $>$

$</$ DataItem $>$

$<$ DataItem category="SAMPLE" id="Mazak03-S2_4" name="S2temp" nativeUnits=" CELSIUS" type="TEMPERATURE" units $=$ "CELSIUS" $/>$

$<$ DataItem category="CONDITION" id="Mazak03-S2_5" name="S2load_cond" type=" LOAD" />

$<$ DataItem category="CONDITION" id="Mazak03-S2_6" name="S2temp_cond" type=" TEMPERATURE" />

$<$ DataItems $>$

$</$ Rotary $>$

$</$ Components $>$

$</$ Axes $>$

<Controller id="Mazak03-controller_1" name="controller">

$<$ DataItems $>$

$<$ DataItem category="CONDITION" id="Mazak03-controller_2" name="comms_cond" type $=$ "COMMUNICATIONS" />

$<$ DataItem category="CONDITION" id="Mazak03-controller_3" name="logic_cond" type="LOGIC_PROGRAM" />

$<$ DataItem category="CONDITION" id="Mazak03-controller_4" name="system_cond" type="SYSTEM" />

$<$ DataItem category="EVENT" id="Mazak03-controller_5" name="pallet_num" type="x 


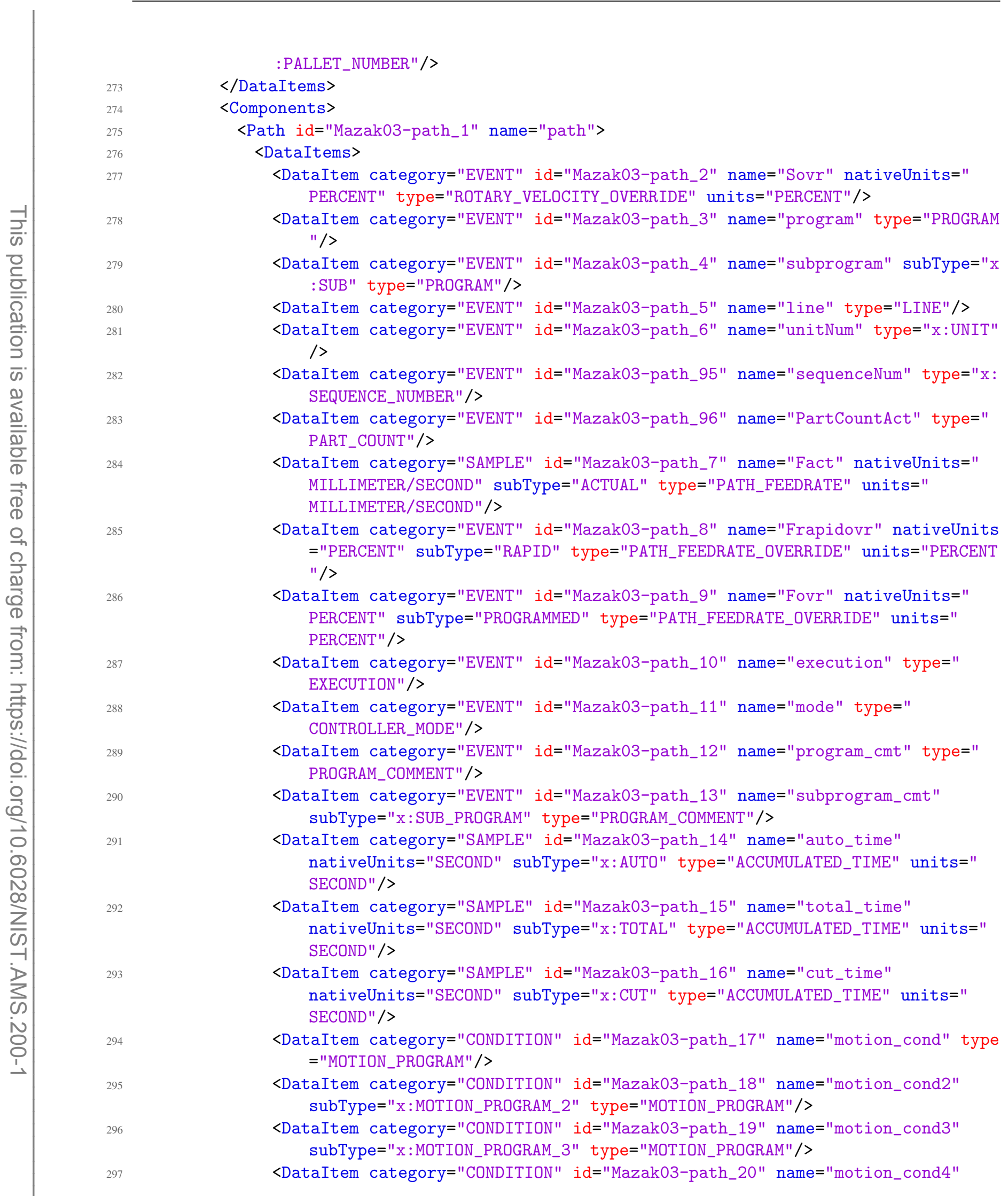

This publication freely available at: https://doi.org/10.6028/NIST.AMS.200-1

Page 22 
subType $=" x:$ MOTION_PROGRAM_4" type="MOTION_PROGRAM" />

<DataItem category="CONDITION" id="Mazak03-path_21" name="path_system" type $="$ SYSTEM" $/>$

<DataItem category="CONDITION" id="Mazak03-path_22" name="path_system2" subType="x:SYSTEM_1" type="SYSTEM"/>

<DataItem category="CONDITION" id="Mazak03-path_23" name="path_system3" subType $=" \mathrm{x}$ :SYSTEM_2" type $=$ "SYSTEM"/>

<DataItem category="CONDITION" id="Mazak03-path_24" name="path_system4" subType="x:SYSTEM_3" type="SYSTEM"/>

$<$ DataItem category="EVENT" id="Mazak03-path_25" name="Tool_number" type=" TOOL_ASSET_ID" />

$<$ DataItem category="EVENT" id="Mazak03-path_26" name="Tool_group" type=" $\mathrm{x}$ : TOOL_GROUP" />

$<$ DataItem category="EVENT" id="Mazak03-path_27" name="Tool_suffix" type=" $\mathrm{x}$ : TOOL_SUFFIX"/>

$<$ DataItems $>$

$</$ Path $>$

$</$ Components $>$

$</$ Controller $>$

<Systems id="Mazak03-systems_1" name="systems">

$<$ Components>

<Electric id="Mazak03-electric_1" name="electric">

$<$ DataItems $>$

$<$ DataItem category="EVENT" id="Mazak03-electric_2" name="power" type=" POWER_STATE"/>

$<$ DataItem category="CONDITION" id="Mazak03-electric_3" name="electric_temp" type="TEMPERATURE" />

$</$ DataItems $>$

$\langle/$ Electric $>$

<Hydraulic id="Mazak03-hydraulic_1" name="hydraulic">

$<$ DataItems $>$

$<$ DataItem category="CONDITION" id="Mazak03-hydraulic_2" name="hydra_cond" type="PRESSURE" />

$</$ DataItems $>$

$</$ Hydraulic $>$

<Coolant id="Mazak03-coolant_1" name="coolant">

$<$ DataItems>

$<$ DataItem category="CONDITION" id="Mazak03-coolant_2" name="coolant_pres" type="PRESSURE" />

$<$ DataItem category="CONDITION" id="Mazak03-coolant_3" name="coolant_temp" type="TEMPERATURE" />

$<$ DataItem category="CONDITION" id="Mazak03-coolant_4" name="coolant_level" type="LEVEL" / >

$</$ DataItems $>$

$</$ Coolant $>$

<Pneumatic id="Mazak03-pneumatic_1" name="pneumatic">

$<$ DataItems $>$

$<$ DataItem category="CONDITION" id="Mazak03-pneumatic_2" name="pneu_cond" type="PRESSURE" />

$</$ DataItems $>$

$<$ Pneumatic $>$ 


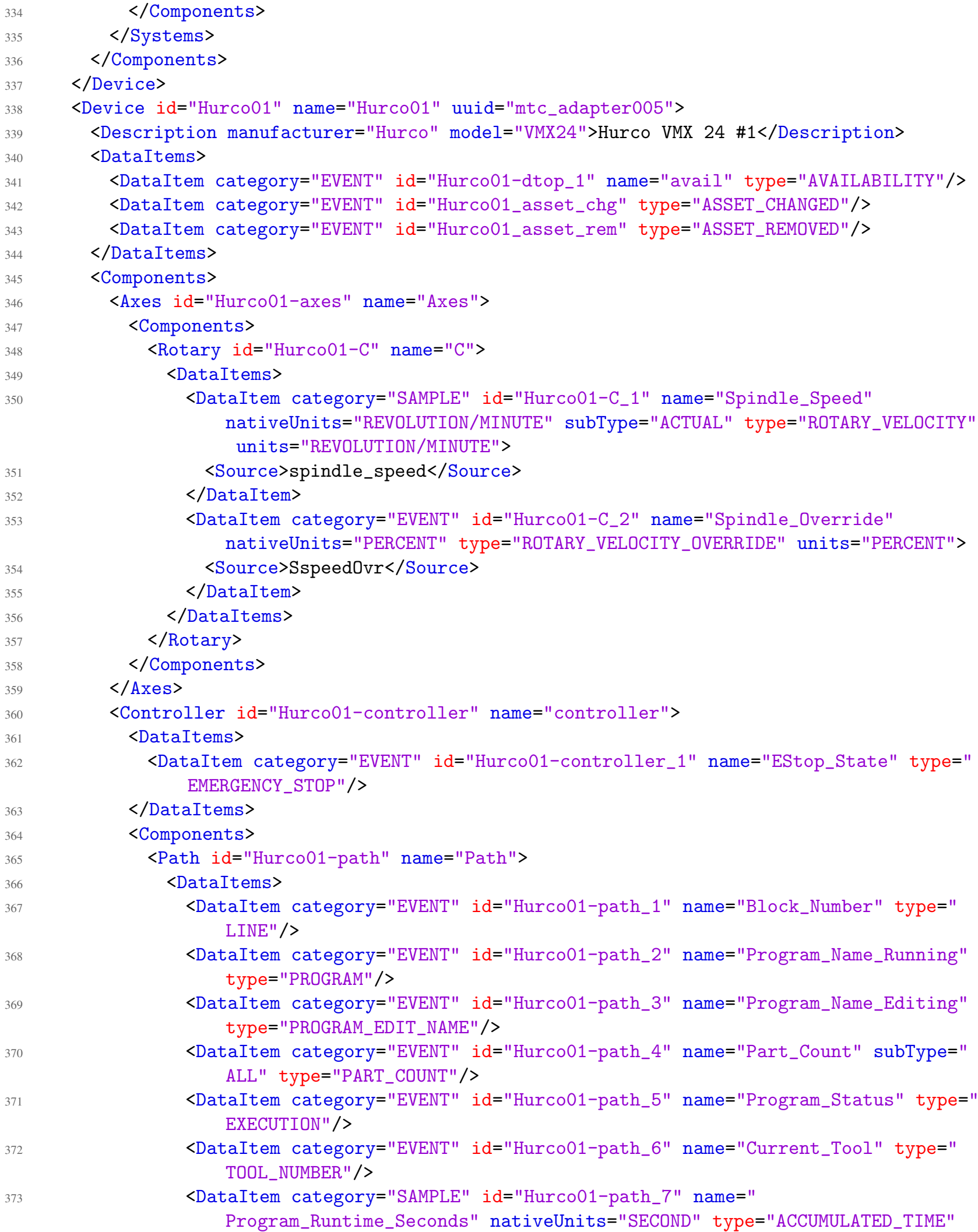


units="SECOND"/>

<DataItem category="SAMPLE" id="Hurco01-path_8" name="Spindle_Time" nativeUnits="SECOND" type="ACCUMULATED_TIME" units="SECOND" /> <DataItem category="SAMPLE" id="Hurco01-path_9" name="Feed_Rate" nativeUnits="MILLIMETER/SECOND" subType="ACTUAL" type="PATH_FEEDRATE" units="MILLIMETER/SECOND" />

$<$ DataItem category="SAMPLE" id="Hurco01-path_10" name="Feed_Override" nativeUnits="PERCENT" type="PATH_FEEDRATE_OVERRIDE" units="PERCENT" $/>$ <DataItem category="SAMPLE" id="Hurco01-path_11" name="Rapid_Override" nativeUnits="PERCENT" subType="RAPID" type="PATH_FEEDRATE_OVERRIDE" units="PERCENT" / >

$</$ DataItems $>$

$</$ Path $>$

$</$ Components $>$

$</$ Controller $>$

$</$ Components $>$

$</$ Device $>$

<Device id="Hurco02" name="Hurco02" uuid="mtc_adapter006">

<Description manufacturer="Hurco" model="VMX24">Hurco VMX 24 \#2</Description>

$<$ DataItems $>$

<DataItem category="EVENT" id="Hurco02-dtop_1" name="avail" type="AVAILABILITY"/>

<DataItem category="EVENT" id="Hurco02_asset_chg" type="ASSET_CHANGED" />

$<$ DataItem category="EVENT" id="Hurco02_asset_rem" type="ASSET_REMOVED" />

$</$ DataItems $>$

$<$ Components $>$

$<$ Axes id="Hurco02-axes" name="Axes" $>$

$<$ Components>

$<$ Rotary id="Hurco02-C" name="C">

$<$ DataItems $>$

<DataItem category="SAMPLE" id="Hurco02-C_1" name="Spindle_Speed" nativeUnits="REVOLUTION/MINUTE" subType="ACTUAL" type="ROTARY_VELOCITY" units="REVOLUTION/MINUTE" >

$<$ Source>spindle_speed</Source>

$</$ DataItem $>$

$<$ DataItem category="EVENT" id="Hurco02-C_2" name="Spindle_Override" nativeUnits="PERCENT" type="ROTARY_VELOCITY_OVERRIDE" units="PERCENT"> $<$ Source>SspeedOvr</Source>

$</$ DataItem $>$

$</$ DataItems $>$

$</$ Rotary $>$

$</$ Components $>$

$</$ Axes $>$

<Controller id="Hurco02-controller" name="controller">

$<$ DataItems $>$

$<$ DataItem category="EVENT" id="Hurco02-controller_1" name="EStop_State" type=" EMERGENCY_STOP"/>

$</$ DataItems $>$

$<$ Components>

$<$ Path id="Hurco02-path" name="Path">

<DataItems

$<$ DataItem category="EVENT" id="Hurco02-path_1" name="Block_Number" type=" 
LINE" />

$<$ DataItem category="EVENT" id="Hurco02-path_2" name="Program_Name_Running" type="PROGRAM" />

<DataItem category="EVENT" id="Hurco02-path_3" name="Program_Name_Editing" type="PROGRAM_EDIT_NAME"/>

$<$ DataItem category="EVENT" id="Hurco02-path_4" name="Part_Count" subType=" ALL" type="PART_COUNT"/>

$<$ DataItem category="EVENT" id="Hurco02-path_5" name="Program_Status" type=" EXECUTION"/>

<DataItem category="EVENT" id="Hurco02-path_6" name="Current_Tool" type=" TOOL_NUMBER"/>

$<$ DataItem category="SAMPLE" id="Hurco02-path_7" name=" Program_Runtime_Seconds" nativeUnits="SECOND" type="ACCUMULATED_TIME" units $=$ "SECOND" />

<DataItem category="SAMPLE" id="Hurco02-path_8" name="Spindle_Time" nativeUnits="SECOND" type="ACCUMULATED_TIME" units="SECOND" />

<DataItem category="SAMPLE" id="Hurco02-path_9" name="Feed_Rate" nativeUnits="MILLIMETER/SECOND" subType="ACTUAL" type="PATH_FEEDRATE" units="MILLIMETER/SECOND" />

$<$ DataItem category="SAMPLE" id="Hurco02-path_10" name="Feed_Override" nativeUnits="PERCENT" type="PATH_FEEDRATE_OVERRIDE" units="PERCENT"/>

<DataItem category="SAMPLE" id="Hurco02-path_11" name="Rapid_Override" nativeUnits="PERCENT" subType="RAPID" type="PATH_FEEDRATE_OVERRIDE" units="PERCENT"/>

$</$ DataItems $>$

$</$ Path $>$

$</$ Components $>$

$</$ Controller $>$

$</$ Components $>$

$</$ Device $>$

<Device id="Hurco03" name="Hurco03" uuid="mtc_adapter007">

<Description manufacturer="Hurco" model="VMX24">Hurco VMX 24 \#3</Description>

$<$ DataItems>

<DataItem category="EVENT" id="Hurco03-dtop_1" name="avail" type="AVAILABILITY"/>

<DataItem category="EVENT" id="Hurco03_asset_chg" type="ASSET_CHANGED"/>

<DataItem category="EVENT" id="Hurco03_asset_rem" type="ASSET_REMOVED"/>

$</$ DataItems $>$

$<$ Components>

<Axes id="Hurco03-axes" name="Axes">

<Components>

<Rotary id="Hurco03-C" name="C">

<DataItems>

<DataItem category="SAMPLE" id="Hurco03-C_1" name="Spindle_Speed" nativeUnits="REVOLUTION/MINUTE" subType="ACTUAL" type="ROTARY_VELOCITY" units="REVOLUTION/MINUTE" $>$

$<$ Source>spindle_speed</Source>

</DataItem>

<DataItem category="EVENT" id="Hurco03-C_2" name="Spindle_Override" nativeUnits="PERCENT" type="ROTARY_VELOCITY_OVERRIDE" units="PERCENT">

$<$ Source>SspeedOvr</Source $>$

$</$ DataItem $>$ 


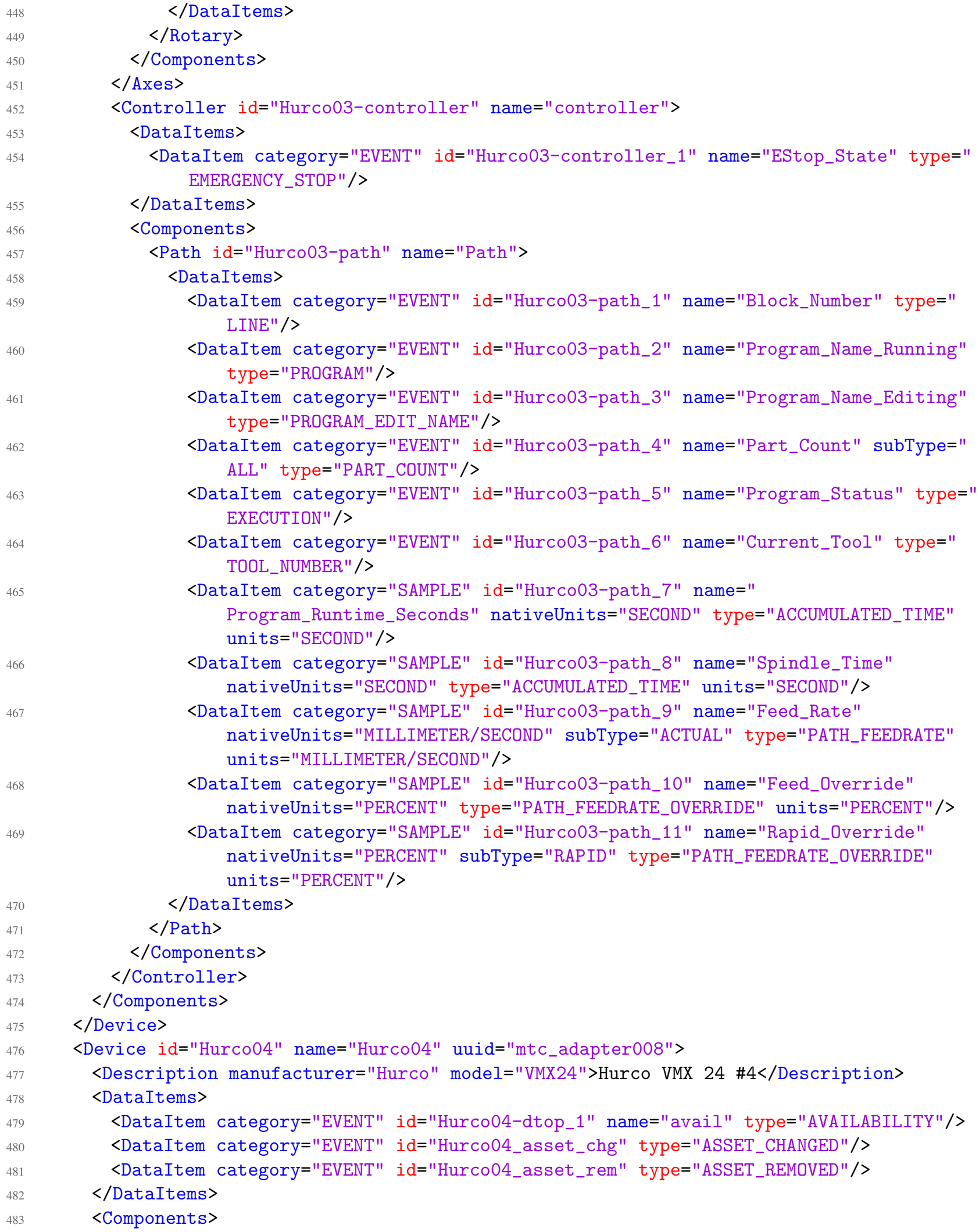




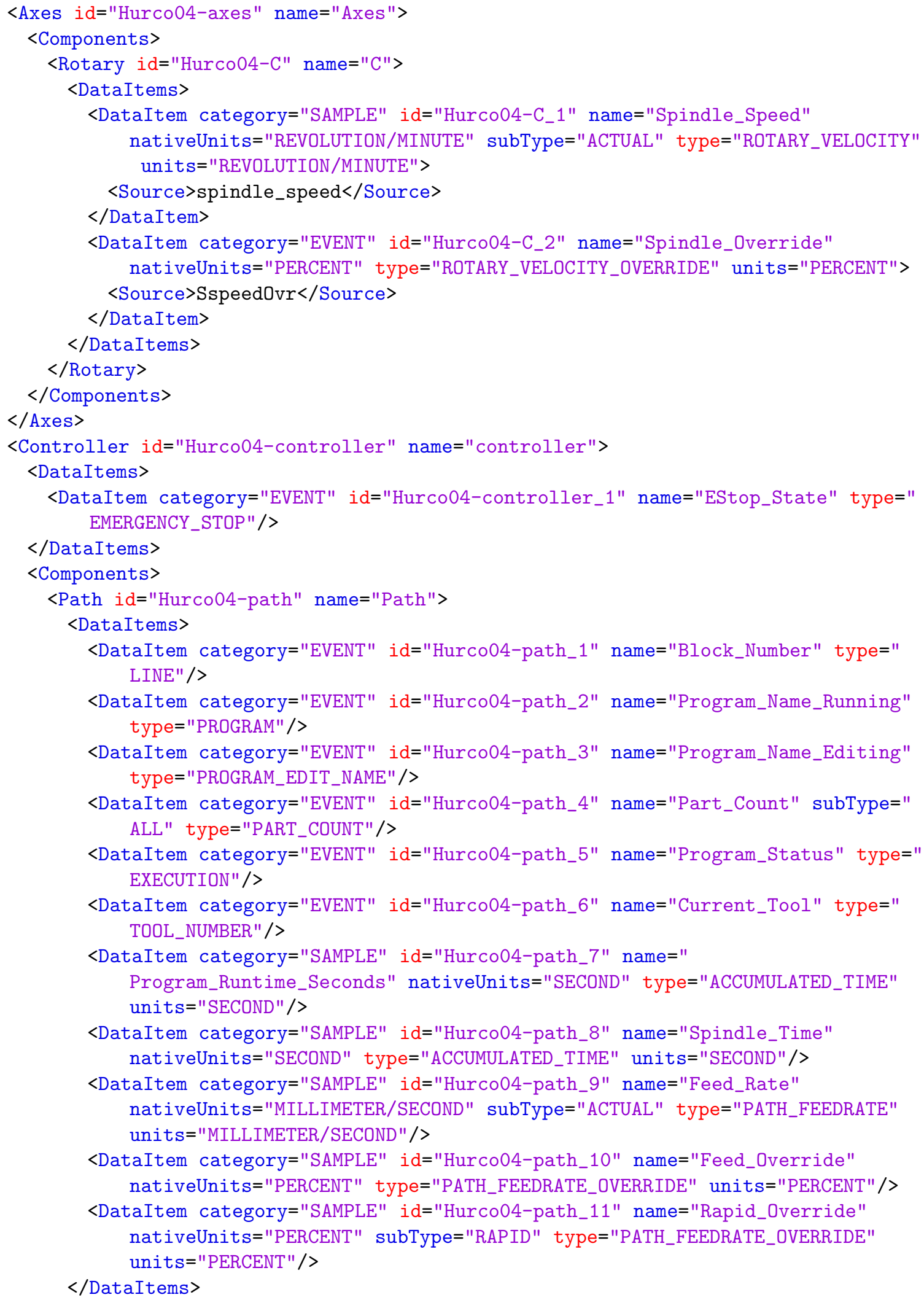




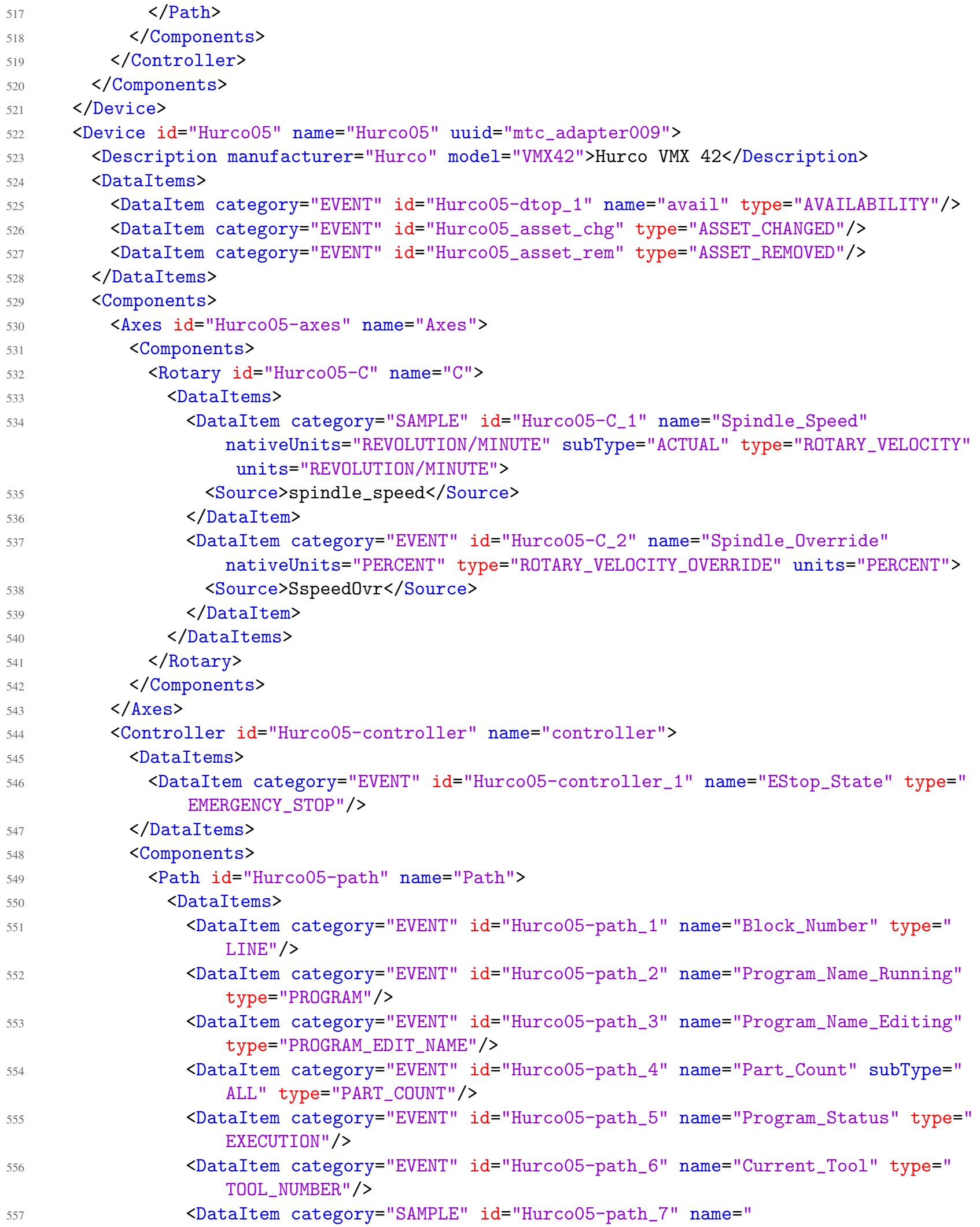


Program_Runtime_Seconds" nativeUnits="SECOND" type="ACCUMULATED_TIME" units="SECOND"/>

<DataItem category="SAMPLE" id="Hurco05-path_8" name="Spindle_Time" nativeUnits="SECOND" type="ACCUMULATED_TIME" units="SECOND" />

<DataItem category="SAMPLE" id="Hurco05-path_9" name="Feed_Rate" nativeUnits="MILLIMETER/SECOND" subType="ACTUAL" type="PATH_FEEDRATE" units="MILLIMETER/SECOND" />

<DataItem category="SAMPLE" id="Hurco05-path_10" name="Feed_Override" nativeUnits="PERCENT" type="PATH_FEEDRATE_OVERRIDE" units="PERCENT"/> <DataItem category="SAMPLE" id="Hurco05-path_11" name="Rapid_Override" nativeUnits="PERCENT" subType="RAPID" type="PATH_FEEDRATE_OVERRIDE" units="PERCENT"/>

$</$ DataItems $>$

$</$ Path $>$

$</$ Components $>$

$</$ Controller $>$

$</$ Components $>$

$</$ Device $>$

<Device id="Hurco06" name="Hurco06" uuid="mtc_adapter010">

<Description manufacturer="Hurco" model="VMX64">Hurco VMX 64</Description>

<DataItems>

<DataItem category="EVENT" id="Hurco06-dtop_1" name="avail" type="AVAILABILITY"/>

<DataItem category="EVENT" id="Hurco06_asset_chg" type="ASSET_CHANGED"/>

<DataItem category="EVENT" id="Hurco06_asset_rem" type="ASSET_REMOVED"/> $</$ DataItems $>$

$<$ Components>

<Axes id="Hurco06-axes" name="Axes">

<Components>

<Rotary id="Hurco06-C" name="C">

<DataItems>

<DataItem category="SAMPLE" id="Hurco06-C_1" name="Spindle_Speed" nativeUnits="REVOLUTION/MINUTE" subType="ACTUAL" type="ROTARY_VELOCITY" units="REVOLUTION/MINUTE" $>$ $<$ Source>spindle_speed</Source>

$</$ DataItem>

<DataItem category="EVENT" id="Hurco06-C_2" name="Spindle_Override" nativeUnits="PERCENT" type="ROTARY_VELOCITY_OVERRIDE" units="PERCENT"> $<$ Source>SspeedOvr</Source>

$</$ DataItem $>$

$</$ DataItems $>$

$</$ Rotary $>$

$</$ Components $>$

$</$ Axes $>$

<Controller id="Hurco06-controller" name="controller">

<DataItems>

<DataItem category="EVENT" id="Hurco06-controller_1" name="EStop_State" type=" EMERGENCY_STOP"/>

$</$ DataItems $>$

$<$ Components>

<Path id="Hurco06-path" name="Path">

$<$ DataItems> 


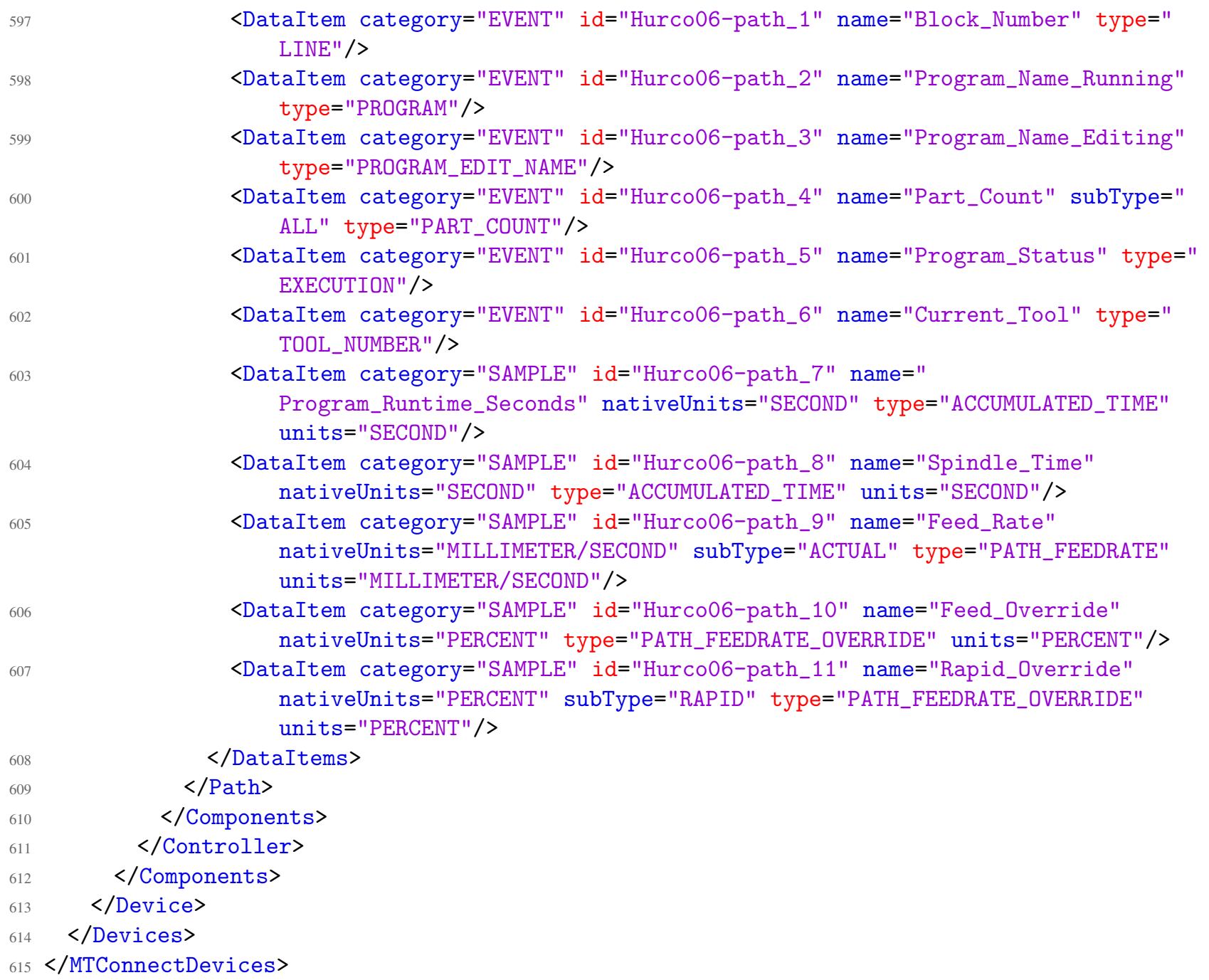

